\title{
A sieve bootstrap test for cointegration in a conditional error correction model
}

Citation for published version (APA):

Palm, F. C., Smeekes, S., \& Urbain, J. R. Y. J. (2007). A sieve bootstrap test for cointegration in a conditional error correction model. METEOR, Maastricht University School of Business and Economics. METEOR Research Memorandum No. 054 https://doi.org/10.26481/umamet.2007054

Document status and date:

Published: 01/01/2007

DOI:

10.26481/umamet.2007054

Document Version:

Publisher's PDF, also known as Version of record

\section{Please check the document version of this publication:}

- A submitted manuscript is the version of the article upon submission and before peer-review. There can be important differences between the submitted version and the official published version of record.

People interested in the research are advised to contact the author for the final version of the publication, or visit the DOI to the publisher's website.

- The final author version and the galley proof are versions of the publication after peer review.

- The final published version features the final layout of the paper including the volume, issue and page numbers.

Link to publication

\footnotetext{
General rights rights.

- You may freely distribute the URL identifying the publication in the public portal. please follow below link for the End User Agreement:

www.umlib.nl/taverne-license

Take down policy

If you believe that this document breaches copyright please contact us at:

repository@maastrichtuniversity.nl

providing details and we will investigate your claim.
}

Copyright and moral rights for the publications made accessible in the public portal are retained by the authors and/or other copyright owners and it is a condition of accessing publications that users recognise and abide by the legal requirements associated with these

- Users may download and print one copy of any publication from the public portal for the purpose of private study or research.

- You may not further distribute the material or use it for any profit-making activity or commercial gain

If the publication is distributed under the terms of Article $25 \mathrm{fa}$ of the Dutch Copyright Act, indicated by the "Taverne" license above, 
Franz C. Palm, Stephan Smeekes, Jean-Pierre Urbain

A Sieve Bootstrap Test for Cointegration in a Conditional Error Correction Model

$\mathrm{RM} / 07 / 054$

JEL code: C15, C32

\section{METE@R}

Maastricht research school of Economics of TEchnology and ORganizations

Universiteit Maastricht

Faculty of Economics and Business Administration P.O. Box 616

NL - 6200 MD Maastricht

phone : ++31433883830

fax : ++31433884873 


\title{
A Sieve Bootstrap Test for Cointegration in a Conditional Error Correction Model
}

\author{
Franz C. Palm Stephan Smeekes* Jean-Pierre Urbain \\ Department of Quantitative Economics \\ Universiteit Maastricht
}

December 14, 2007

\begin{abstract}
In this paper we propose a bootstrap version of the Wald test for cointegration in a single-equation conditional error correction model. The multivariate sieve bootstrap is used to deal with dependence in the series. We show that the introduced bootstrap test is asymptotically valid.

We also analyze the small sample properties of our test by simulation and compare it with the asymptotic test and several alternative bootstrap tests. The bootstrap test offers significant improvements in terms of size properties over the asymptotic test, while having similar power properties. It also performs at least as well as the alternative bootstrap tests considered in terms of size and power.

The sensitivity of the bootstrap test to the allowance for deterministic components is also investigated. Simulation results show that the tests with sufficient deterministic components included are insensitive to the true value of the trends in the model, and retain correct size.
\end{abstract}

JEL Classification: C15, C32.

Keywords: sieve bootstrap, cointegration, error correction model.

\section{Introduction}

In this paper we present a bootstrap version of the single-equation error correction model (ECM) Wald test for cointegration originally proposed by Boswijk (1994). ECM tests are

\footnotetext{
${ }^{*}$ Corresponding author: Department of Quantitative Economics, Universiteit Maastricht, P.O. Box 616, 6200 MD Maastricht, The Netherlands. Email: S.Smeekes@ke.unimaas.nl. Previous versions of this paper have been presented at an Ente Luigi Einaudi Seminar in Econometrics in Rome, November 2007, and at the first workshop of the Methods in International Finance Network in Maastricht, September 2007. We gratefully acknowledge the comments by participants at these seminars. The usual disclaimer applies.
} 
an attractive option for cointegration testing, as contrary to the more popular Engle and Granger (1987) type of ADF tests, ECM tests do not suffer from imposing invalid common factor restrictions (Kremers, Ericsson, and Dolado, 1992; Banerjee, Dolado, and Mestre, 1998; Zivot, 2000). Moreover, Pesavento (2004) analyzes several tests which have as null hypothesis no cointegration, including the residual ADF test by Engle and Granger (1987) and the maximum eigenvalue test by Johansen and Juselius (1990), and finds that among these the ECM tests perform best in terms of power both in small and large samples, while performing similarly as the other tests in terms of size.

The ECM Wald test has as main advantage over the ECM t-test (Banerjee et al., 1998) that it is more intuitive and one does not have to add a redundant regressor if no particular cointegrating vector is specified. Although the Wald ECM test performs well in general, especially in terms of power, it still suffers from size distortions in finite samples. It is well known that the bootstrap's ability to provide asymptotic refinements often leads to a reduction of size distortions for hypothesis tests. Even under "non-favorable" conditions for the bootstrap, under which it is unclear whether it provides asymptotic refinements, such as when dealing with nonstationary time series, the bootstrap has been shown to reduce size distortions in finite samples (see for example the tests for unit roots considered in Palm, Smeekes, and Urbain, 2007).

Little is known so far about the application of the bootstrap to cointegration testing in error correction models. Swensen (2006) and Trenkler (2006) provide theoretical and simulation results on bootstrap versions of the trace test for cointegration rank by Johansen (1995). Their setting differs from ours in that we a priori assume the cointegrating rank is at most one. Seo (2006) provides analytical and simulation results for a residual-based bootstrap test in a threshold vector error correction model. Closer to our setting, Mantalos and Shukur (1998) and Ahlgren (2000) consider a bootstrap version of the test with known cointegrating vector by Kremers et al. (1992), however they only provide simulation results for a simple model. In this paper we will allow for more general dependence over time in our model, and we provide analytical as well as simulation results.

We use in this paper the sieve bootstrap introduced by Bühlmann (1997), a method that can handle time series dependence in the form of a general linear process by approximating it as an autoregressive process. The sieve bootstrap method is easy to use and performs well relative to other time series bootstrap methods, especially the block bootstrap (for a comparison between methods in the unit root setting, see Palm et al., 2007). The condition of linearity is fulfilled by a large class of processes, and is needed to validate the use of the Wald test without the bootstrap as well.

The contribution of the paper is threefold. First, we prove that the sieve bootstrap version of the single-equation Wald test of no cointegration is asymptotically valid. The proofs are given in detail for the multivariate setting, such that proofs of other types of tests could be 
done along the same lines as presented here. Second, we provide simulation results showing that the bootstrap version of the Wald test has better properties in finite samples than the asymptotic test. Third, we investigate the sensitivity of the bootstrap to allowing for deterministic components.

The structure of the paper is as follows. Section 2 explains the model and assumptions. The construction of the bootstrap test and the establishment of its asymptotic validity are discussed in Section 3. Our simulation study is presented in Section 4. The inclusion of deterministic components is discussed in Section 5. Section 6 concludes. All proofs are contained in Appendix A.

Finally, a word on notation. We use $|\cdot|$ to denote the Euclidean norm for vectors and matrices, i.e. $|v|=\left(v^{\prime} v\right)^{1 / 2}$ for a vector $v$ and $|M|=\left(\operatorname{tr} M^{\prime} M\right)^{1 / 2}$ for a matrix $M$. For matrices we also use the operator norm $\|M\|=\max _{v}|M v| /|v| . \quad W(r)=\left(W_{1}(r), W_{2}(r)^{\prime}\right)^{\prime}$ denotes a multivariate standard Brownian motion of dimension $(1+l) .[x]$ is the largest integer smaller than or equal to $x$. Convergence in distribution (probability) is denoted by $\stackrel{d}{\rightarrow}$ $(\stackrel{p}{\rightarrow})$. Bootstrap quantities (conditional on the original sample) are indicated by appending a superscript $*$ to the standard notation. Subscripts $p$ (or $q$ ) are used to indicate quantities depending on approximations of infinite order models by models of order $p$ (or $q$ ). For simplicity we suppress these subscripts whenever clarity allows it.

\section{The model}

Our Data Generating Process (DGP) is closely related to that of Pesavento (2004). We let our $(1+l)$-dimensional time series $z_{t}=\left(y_{t}, x_{t}^{\prime}\right)^{\prime}$ be described by the process

$$
z_{t}=\mu+\tau t+\zeta_{t}
$$

The stochastic component $\zeta_{t}$ is given by

$$
\Delta \zeta_{t}=(\rho-1) \alpha \beta^{\prime} \zeta_{t-1}+u_{t}
$$

where

$$
u_{t}=\Psi(L) \varepsilon_{t}
$$

with $\Psi(z)=\sum_{j=}^{\infty} \Psi_{j} z^{j}$. Furthermore we assume that $\zeta_{0}=0 .{ }^{1}$ Under our null hypothesis $H_{0}: \rho=1$ there is no cointegration. Under our alternative $H_{1}: \rho<1$ there is cointegration with a single cointegrating vector $\beta$ and the error correction term must be present in the equation for $y_{t}$. Also, we impose $\alpha_{1}=1$ and $\alpha_{2}=0$, which follows from the triangular

\footnotetext{
${ }^{1}$ This assumption is made for expositional simplicity only and can be extended to $\zeta_{0}=O_{p}(1)$.
} 
representation of the model as in Pesavento (2004) and is needed for identification purposes. ${ }^{2}$ These points are formalized in Assumption 1.

Assumption 1. We assume

(i) $\alpha \beta^{\prime}$ is of rank 1, i.e. there is a single $(1+l)$-dimensional cointegrating vector $\beta$,

(ii) $\beta$ is normalized on the coefficient of $y_{t}$, i.e. $\beta=\left(1,-\gamma^{\prime}\right)^{\prime}$,

(iii) $\alpha=\left(1,0^{\prime}\right)^{\prime}$.

Equation (3) shows that we take $u_{t}$ to be a linear process (Phillips and Solo, 1992). Assumption 2 ensures the invertibility of $u_{t}$ and the existence of moments of $\varepsilon_{t}$. These assumptions are not very stringent and encompass many assumptions (including all finite ARMA models) that are often used in cointegration analysis.

Assumption 2. We assume

(i) $\varepsilon_{t}$ are i.i.d. with $\mathrm{E}\left(\varepsilon_{t}\right)=0, \mathrm{E}\left(\varepsilon_{t} \varepsilon_{t}^{\prime}\right)=\Sigma$ and $\mathrm{E}\left|\varepsilon_{t}\right|^{4}<\infty$.

(ii) $\operatorname{det}(\Psi(z)) \neq 0$ for all $|z| \leq 1$, and $\sum_{j=0}^{\infty} j\left|\Psi_{j}\right|<\infty$.

By Assumption 2 we may write $\Phi(L)=\sum_{j=0}^{\infty} \Phi_{j} L^{j}=\Psi(L)^{-1}$. Then we may substitute equation (1) into (2) and apply the Beveridge-Nelson decomposition to show as in Pesavento (2004) that this model can be rewritten in VECM form

$$
\Delta z_{t}=(\rho-1) \Phi(1) \alpha \beta^{\prime}\left(z_{t-1}-\mu-\tau(t-1)\right)+\tilde{\tau}+\Phi^{*}(L) \Delta z_{t-1}+\varepsilon_{t}
$$

where

$$
\Phi^{*}(L)=\sum_{j=0}^{\infty}\left((1-\rho)\left(\sum_{i=j+1}^{\infty} \Phi_{i}\right) \alpha \beta^{\prime}-\Phi_{j+1}\right) L^{j}
$$

and

$$
\tilde{\tau}=\left(\sum_{j=0}^{\infty} \Phi_{j}+(\rho-1)\left(\sum_{j=0}^{\infty} \sum_{i=j+1}^{\infty} \Phi_{i}\right) \alpha \beta^{\prime}\right) \tau .
$$

It can be seen from the above representation that $z_{t}$ has a drift if $\tau \neq 0$, and this drift leads to a linear trend in the cointegrating relation if $\beta^{\prime} \tau \neq 0$. The constant $\mu$ only appears in the cointegrating relation; note that the cointegrating relation has mean zero if $\beta^{\prime} \mu=0$.

\footnotetext{
${ }^{2}$ Pesavento (2004) shows that this restriction corresponds to the assumption that $x_{t}$ are not mutually cointegrated, as required under Assumption 1(i), and are known a priori to be $I(1)$.
} 
Pesavento shows the model can be written in triangular form as well, which makes it a very flexible model. As we do not need that representation here, we continue on the VECM representation (4) and condition on $x_{t}$ to obtain

$$
\Delta y_{t}=(\rho-1) \theta \beta^{\prime}\left(z_{t-1}-\mu-\tau(t-1)\right)+\tilde{\tau}_{1} t+\pi_{0}^{\prime} \Delta x_{t}+\sum_{j=1}^{\infty} \pi_{j}^{\prime} \Delta z_{t-1}+\xi_{t}
$$

where $^{3} \xi_{t} \sim$ i.i.d. $\left(0, \omega^{2}\right)$ and $\theta=\Phi_{1}(1) \alpha-\Sigma_{12} \Sigma_{22}^{-1} \Phi_{2}(1) \alpha$ with $\Phi(1)=\left(\Phi_{1}(1)^{\prime}, \Phi_{2}(1)^{\prime}\right)^{\prime}$.

The advantage of this framework is that its assumptions are weaker than what is usually assumed for tests based on a conditional ECM, as it does not impose that $x_{t}$ are weakly exogenous for $\beta$ under the alternative of cointegration. Under the null however, the error correction term does not appear in the marginal equations, which makes a test on the error correction term in the conditional model a valid test for cointegration (Boswijk, 1994).

\section{$3 \quad$ The bootstrap test and asymptotics}

\subsection{Test statistic}

The Wald test proposed by Boswijk (1994) is based on the conditional model (5). Consider the regression

$$
\Delta y_{t}=\delta^{\prime} \tilde{z}_{t-1}+\lambda^{\prime} D_{t}+\pi_{0}^{\prime} \Delta x_{t}+\sum_{j=1}^{p} \pi_{j}^{\prime} \Delta z_{t-1}+\xi_{p, t}
$$

where $D_{t}$ are the (unrestricted) deterministic components included in the regression and $\tilde{z}_{t-1}=\left(z_{t-1}^{\prime}, D_{t-1}^{r}\right)^{\prime}$, where $D_{t}^{r}$ are the deterministic components that are restricted to be equal to zero under the null (see Section 5). If $\rho=1, \delta^{\prime}=(\rho-1) \theta \beta^{\prime}=0$, which leads us to the test statistic

$$
T_{\text {wald }}=\hat{\delta}^{\prime} \widehat{\operatorname{Var}(\hat{\delta})}^{-1} \hat{\delta}
$$

where $\hat{\delta}$ is the OLS estimator of $\delta$ in $(6)$ and $\widehat{\operatorname{Var}(\hat{\delta})}$ is its estimated covariance matrix. The null hypothesis of no cointegration is then rejected for large values of $T_{\text {wald }}$.

We let the lag length $p$ in regression (6) grow to infinity at a controlled rate.

Assumption 3. Let $p \rightarrow \infty$ and $p=o\left(n^{1 / 2}\right)$ as $n \rightarrow \infty$.

The limiting distribution of $T_{\text {wald }}$ can be found in Boswijk (1994) for the ECM with finite autoregressive dependence and in Pesavento (2004) for the infinite-order model. The asymptotic distribution of the test without the inclusion of any deterministic components (and with $\mu=\tau=0$ ) is given for completeness in Lemma 1 without proof.

\footnotetext{
${ }^{3} \omega^{2}=\sigma_{11}-\Sigma_{12} \Sigma_{22}^{-1} \Sigma_{21}$, where $\Sigma$ has been partitioned conformably with $y_{t}$ and $x_{t}$.
} 
Lemma 1. Under Assumptions 2 and 3 we have that

$$
T_{\text {wald }} \stackrel{d}{\rightarrow} \int_{0}^{1} d W_{1}(r) W(r)^{\prime}\left[\int_{0}^{1} W(r) W(r)^{\prime} d r\right]^{-1} \int_{0}^{1} W(r) d W_{1}(r)
$$

where $T_{\text {wald }}$ is defined in equation (7).

\subsection{Bootstrap method}

The multivariate sieve bootstrap method we employ here is very similar to the one employed by Chang, Park, and Song (2006) for the estimation of cointegrating vectors. The full algorithm is given below.

\section{Bootstrap Algorithm.}

Step 1: Fit a $\operatorname{VAR}(q)$ process to $\Delta z_{t}$ by OLS and save the residuals

$$
\hat{\varepsilon}_{q, t}=\Delta z_{t}-\hat{\lambda}_{s} D_{t}^{s}-\sum_{j=1}^{q} \hat{\Phi}_{j} \Delta z_{t-j}
$$

where $D_{t}^{s}$ are the deterministic components included in this sieve estimation (see Section 5 for details). Recenter the residuals $\hat{\varepsilon}_{q, t}$ in the case where no constant is included to eliminate any drifts in the resampled series and save the recentered residuals $\tilde{\varepsilon}_{q, t}=\hat{\varepsilon}_{q, t}-(n-q-1)^{-1} \sum_{t} \hat{\varepsilon}_{q, t} \cdot{ }^{4}$

Step 2: Resample with replacement from $\tilde{\varepsilon}_{q, t}$ to obtain bootstrap errors $\varepsilon_{t}^{*}$.

Step 3: Build $u_{t}^{*}$ recursively as

$$
u_{t}^{*}=\sum_{j=1}^{q} \hat{\Phi}_{j} u_{t-j}^{*}+\varepsilon_{t}^{*}
$$

using the estimated parameters $\hat{\Phi}_{j}$ from Step 1 , and build $z_{t}^{*}$ as

$$
z_{t}^{*}=z_{t-1}^{*}+u_{t}^{*}
$$

Note that it is unnecessary to include deterministic components in this step, as the tests we consider are asymptotically similar (see Remark 8 in Section 5).

\footnotetext{
${ }^{4}$ In the cases where we do not include a constant in this regression the residuals may have a sample mean unequal to zero, even though their theoretical mean is zero. As the sample mean of the residuals becomes the population mean of the bootstrap errors, this may lead to (unwanted) drifts in the bootstrap sample.
} 
Step 4: Using the bootstrap sample $z_{t}^{*}$, estimate $\hat{\delta}^{*}$ from the regression

$$
\Delta y_{t}^{*}=\delta^{* \prime} \tilde{z}_{t-1}^{*}+\lambda^{* \prime} D_{t}^{*}+\pi_{0}^{* \prime} \Delta x_{t}^{*}+\sum_{j=1}^{p^{*}} \pi_{j}^{* \prime} \Delta z_{t-1}^{*}+\xi_{p^{*}, t}^{*}
$$

where $\tilde{z}_{t-1}^{*}=\left(z_{t-1}^{* \prime}, D_{t-1}^{r *}\right)^{\prime}$, and calculate the bootstrap test statistic

$$
T_{\mathrm{wald}}^{*}=\hat{\delta}^{* \prime}{\widehat{\operatorname{Var}}{ }^{*}\left(\hat{\delta}^{*}\right)}^{-1} \hat{\delta}^{*}
$$

$D_{t}^{*}$ and $D_{t}^{r *}$ are the bootstrap counterparts of $D_{t}$ and $D_{t}^{r}$. In order to get the correct asymptotic bootstrap distribution, one should always take $D_{t}^{*}=D_{t}$ and $D_{t}^{r *}=D_{t}^{r}$.

Step 5: Repeat Steps 2 to $4 B$ times, obtaining bootstrap test statistics $T_{\text {wald }}^{* b}, b=1, \ldots, B$, and select the bootstrap critical value $c_{\alpha}^{*}$ as $c_{\alpha}^{*}=\min \left\{c: \sum_{b=1}^{B} I\left(T_{\text {wald }}^{* b}>c\right) \leq \alpha\right\}$, or equivalently as the $(1-\alpha)$-quantile of the ordered $T_{\text {wald }}^{* b}$ statistics. Reject the null of no cointegration if $T_{\text {wald }}$, calculated from equations (6) and (7), is larger than $c_{\alpha}^{*}$, where $\alpha$ is the nominal level of the test.

We need to allow the lag length $q$ in the sieve bootstrap to go to infinity at a controlled rate. We will use two assumptions.

Assumption 4. Let $q \rightarrow \infty$ and $q=o\left((n / \ln n)^{1 / 2}\right)$ as $n \rightarrow \infty$.

Assumption $4^{\prime}$. Let $q \rightarrow \infty$ and $q=o\left((n / \ln n)^{1 / 3}\right)$ as $n \rightarrow \infty$.

Remark 1. In Step 3 we need to initialize $u_{t}^{*}$ in (9) and $z_{t}^{*}$ in (10). We propose to generate a large number of values of $u_{t}^{*}$ and delete the first generated values. This will ensure that $u_{t}^{*}$ is a stationary process. The initial values in (9) will then become unimportant as the realization of $u_{t}^{*}$ will not depend on them; hence they may be set equal to zero. An alternative is take to first $q$ values of $u_{t}^{*}$ equal to the first $q$ values of $u_{t}$; this however does not ensure stationarity of $u_{t}^{*}$.

As asymptotically the effect of $z_{0}^{*}$ disappears, we simply set $z_{0}^{*}=0$. The logical alternative here would be to set $z_{0}^{*}=z_{0}$.

Remark 2. Instead of estimating the sieve under the null of no cointegration (which we impose by fitting the VAR model to $\Delta z_{t}$ in Step 1), we may also estimate it under the alternative of cointegration. In this case we would estimate the residuals as

$$
\hat{\varepsilon}_{q, t}=\Delta z_{t}-\hat{\lambda}_{b} D_{t}^{s, a}-\hat{\Phi}_{0} z_{t-1}-\sum_{j=1}^{q} \hat{\Phi}_{j} \Delta z_{t-j},
$$

where $\hat{\Phi}_{0}$ denotes the unrestricted OLS estimator and $D_{t}^{s, a}$ are the deterministic components included in this alternative-based sieve estimation. Note that even for the same deterministic 
setting, $D_{t}^{s, a}$ is not necessarily the same as $D_{t}^{s}$ in (8), as is explained in Section 5 (Remark 10).

In the context of unit root testing, Paparoditis and Politis (2005) advocate the use of such a "residual-based" estimation as opposed to the "difference-based" estimation in (8), claiming that the residual-based tests have better power properties. We will return to this point in our simulations in Section 4.

Remark 3. A second alternative bootstrap strategy would be to base the sieve bootstrap on the conditional/marginal ECM model instead of the VECM/VAR model. In this case we would need two separate equations to estimate residuals in Step 1. We would estimate the residuals from the conditional model as

$$
\hat{\varepsilon}_{1, q, t}=\Delta y_{t}-\hat{\lambda}_{s, 1} D_{t, 1}^{s}-\hat{\pi}_{0}^{\prime} \Delta x_{t}-\sum_{j=1}^{q} \hat{\pi}_{j} \Delta z_{t-j}
$$

and the residuals from the marginal model as

$$
\hat{\varepsilon}_{2, q, t}=\Delta x_{t}-\hat{\lambda}_{s, 2} D_{t, 2}^{s}-\sum_{j=1}^{q} \hat{\Phi}_{2, j} \Delta z_{t-j}
$$

for the difference-based alternative. We can of course also construct a residual-based version of this test. In the simulations in Section 4 we will look at these alternatives as well.

Although such an approach is closer in spirit to the single-equation Wald test statistic, it is basically just a reparametrization of the VECM approach, as the model on which the bootstrap is based is still completely specified. An alternative approach, which would be "truly conditional" on $x_{t}$, is to take $x_{t}$ as fixed and only resample $y_{t}$. To justify such an approach we would have to assume strong exogeneity, see Van Giersbergen and Kiviet (1996) for a discussion. This last approach will not be investigated in this paper.

Remark 4. Although estimation under the alternative is an option in Step 1, it is not possible to build the bootstrap sample $z_{t}^{*}$ in Step 3 based on the alternative hypothesis, i.e. using

$$
z_{t}^{*}=\left(I+\hat{\Phi}_{0}\right) z_{t-1}^{*}+u_{t}^{*}
$$

Basawa, Mallik, McCormick, Reeves, and Taylor (1991) show that if such an alternativebased recursion is used in the unit root setting, the limiting distribution of the bootstrap test statistic is random due to the discontinuity of the limiting distribution at the unit root. The same logic applies here, therefore the null hypothesis of no cointegration must be imposed in Step 3.

Remark 5. To obtain the theoretical results in the next subsection, we set all deterministic components equal to zero, both in the model ( $\mu$ and $\tau$ ) and in the test (all variants of $D_{t}$ ). In Section 5 we will go into more detail about the inclusion of deterministic components, and 
present some simulation results. We conjecture that asymptotic validity still holds in the presence of deterministic components.

Remark 6. In Step 4 we specify the lag length in the bootstrap test regression (11) as $p^{*}$, in order to emphasize that this lag length does not have to be the same as the lag length in the original test regression (6). In finite samples the performance of the bootstrap test will be better if the lag length is allowed to be different. Just as for the original test regression (and the sieve bootstrap), the lag length can be chosen in practice by information criteria like AIC and BIC.

Obviously, $p^{*}$ has to fulfil the same conditions as $p$. Therefore, we can write $p^{*}$ as $p$ in the theoretical results, which is done for notational simplicity.

Remark 7. As we will see in the next subsection, Assumption 4 is sufficient to prove Theorem 1. However, there is one element in the proof of Theorem 2 that is based on the revised version of Park (2002, Proof of Theorem 3.3), which requires the stronger Assumption 4'.

\subsection{Asymptotic results}

In this section we will give the main theoretical results needed to show the asymptotic validity of the bootstrap test. As stated in Remark 5, we derive these results for the tests (and DGP) without deterministic components. The proofs of all the results here plus additional lemmas can be found in Appendix A. Most of the proofs are based on the proofs in Chang et al. (2006), and the papers they refer to.

As we present all our proofs for vector processes, the theory employed in the paper can be used to prove validity of other multivariate bootstrap procedures as well. Note that all our bootstrap weak convergence results hold in probability as we derive all underlying results in probability.

The first step in proving the asymptotic validity is the development of an invariance principle for the bootstrap errors $\varepsilon_{t}^{*}$.

Theorem 1. Under Assumptions 2 and 4, we have that

$$
W_{n}^{*}(r)=n^{-1 / 2} \sum_{t=1}^{[n r]} \varepsilon_{t}^{*} \stackrel{d^{*}}{\longrightarrow} L W(r) \quad \text { in probability }
$$

where $L$ is a $(1+l) \times(1+l)$-dimensional lower triangular matrix such that the Cholesky decomposition of $\Sigma$ is equal to $L L^{\prime}$.

We can show this result by first showing that $\mathrm{E}^{*}\left|\varepsilon_{t}^{*}\right|^{a}=O_{p}(1)$ for some $a>2$, and then referring to Einmahl (1987), who shows that if this condition holds we can show that an invariance principle holds.

From this result, with the help of the Beveridge-Nelson decomposition, we can construct an invariance principle for $u_{t}^{*}$. 
Theorem 2. Under Assumptions 2 and $4^{\prime}$ we have that

$$
B_{n}^{*}(r)=n^{-1 / 2} \sum_{t=1}^{[n r]} u_{t}^{*} \stackrel{d^{*}}{\longrightarrow} B(r) \quad \text { in probability }
$$

where $B(r)$ is a $(1+l)$-dimensional Brownian motion such that $B(r)=\Psi(1) L W(r)$.

Then, using Theorem 2, we can derive the limiting distributions of the elements of the test statistic, and finally show the consistency of the bootstrap variance estimator. With these results, we can then present Theorem 3 which shows the asymptotic distribution of the bootstrap test statistic.

Theorem 3. Under Assumptions 2, 3 and $4^{\prime}$ we have that

$$
T_{\text {wald }}^{*} \stackrel{d^{*}}{\longrightarrow} \int_{0}^{1} d W_{1}(r) W(r)^{\prime}\left[\int_{0}^{1} W(r) W(r)^{\prime} d r\right]^{-1} \int_{0}^{1} W(r) d W_{1}(r) \quad \text { in probability }
$$

where $T_{\text {wald }}^{*}$ is defined in equation (12).

Note that Theorem 3 shows that the bootstrap test statistic has the same asymptotic distribution as the original test statistic, which shows that the bootstrap test is asymptotically valid. Also note that the limiting distribution of the test statistic is asymptotically pivotal, which means that the bootstrap may offer asymptotic refinements, although this does not have to be so.

\section{Simulations}

We wish to study the small sample properties of our test by simulation. We do not only compare our test with the test based on asymptotic critical values (provided by Boswijk, 1994), but also with the three alternative bootstrap tests mentioned in Remarks 2 and 3. We write our bootstrap test in the tables as $T_{v, n}^{*}$, where the subscript $v$ stands for estimation based on the VAR/VECM model, and the $n$ for estimation of the sieve bootstrap under the null. The alternative test discussed in Remark 2 is denoted by $T_{v, a}^{*}$, with the subscript $a$ indicating estimation of the sieve bootstrap under the alternative. Similarly, the two alternatives discussed in Remark 3 are given as $T_{c, n}^{*}$ and $T_{c, a}^{*}$, where the subscript $c$ makes clear that these are based on the conditional/marginal model. Finally, the asymptotic test is denoted as $T_{a s}$.

For our small sample simulation study we use the same setup as Pesavento (2004). We let the bivariate series $\left(y_{t}, x_{t}\right)^{\prime}$ be generated from the triangular system

$$
\begin{aligned}
y_{t} & =\gamma x_{t}+u_{t}, \\
u_{t} & =\rho u_{t-1}+v_{1 t}, \\
\Delta x_{t} & =v_{2 t} .
\end{aligned}
$$


We take $\rho=1$ to analyze the size of tests, and $\rho<1$ for the power. As we are interested in local power, we set $\rho=1+c / n$, where for the sample size we take $n=50$ and $n=100$. The tests are invariant to the true value of $\gamma$ as long as it is non-zero, therefore we set $\gamma=1$. Furthermore we set $u_{0}=x_{0}=0$.

We generate the errors $v_{t}=\left(v_{1 t}, v_{2 t}\right)^{\prime}$ as

$$
(1-\Phi L) v_{t}=(1+\Theta L) \varepsilon_{t}
$$

where $\varepsilon_{t}$ is generated from a bivariate normal distribution with covariance matrix

$$
\Sigma=\left[\begin{array}{ll}
1 & r \\
r & 1
\end{array}\right] .
$$

The exact parameter combinations considered are summarized in Table 1.

\section{INSERT TABLE 1 ABOUT HERE}

We select lag lengths in (6), (8) and (11) by BIC, with maximum lag lengths of 8 for $n=50$ and 11 for $n=100$. Each generated sample is used to perform all the tests on, such that the lag length $p$ in (6) is always the same for all tests. Our results are based on 2000 simulations, with 999 bootstrap replications per simulation.

The results for the DGPs with white noise errors $(\Phi=\Theta=0)$ are given in Table 2. For this case, the asymptotic test has a reasonably good size, but still the bootstrap tests clearly have even better sizes, especially for $n=50$. The rejection frequencies of the bootstrap tests are somewhat less than those of the asymptotic test under the alternatives considered, but it is difficult to compare powers as sizes are not equal. In order to be able to compare powers more clearly, we also give size-corrected powers for the asymptotic test (given in the Table as $\left.T_{s c}\right) .{ }^{5}$ We see that the size-corrected power of the asymptotic test is close to the power of the bootstrap tests, which shows that the higher raw power of the asymptotic test is mainly due to the higher size distortions. All bootstrap tests perform similarly both in terms of size and power, indicating that in this setting there is no evidence for power problems for the difference-based tests.

\section{INSERT TABLE 2 ABOUT HERE}

Table 3 gives the results for the size of the tests for DGPs with autoregressive and movingaverage errors. For all DGPs considered here, there is a very clear advantage of using the bootstrap, which virtually eliminates all size distortions except for the negative movingaverage coefficients. Again note that the difference between the bootstrap and asymptotic

\footnotetext{
${ }^{5}$ There is no need to correct the power of the bootstrap tests, as they have virtually no size distortions; their size-corrected powers would be almost the same as their raw powers.
} 
test is the largest for $n=50$. The bootstrap tests perform fairly similar, with a minor advantage for the difference-based tests, which is especially noticeable for the DGP with negative moving-average coefficients.

\section{INSERT TABLE 3 ABOUT HERE}

To illustrate the power properties for DGPs with some dependence in the errors, we have selected one DGP with autoregressive and one with moving-average coefficients from the set considered above. The results are given in Table 4. Again we have to be careful with comparing raw powers, as sizes of the tests are not equal. We see that the asymptotic test has somewhat higher rejection frequencies than the bootstrap tests, but as in Table 2 the differences correspond to high size distortions of the asymptotic test. This is again confirmed by the size-corrected power of the asymptotic test, which is no better, and in some cases considerably worse, than the power of the bootstrap tests. The difference-based tests appear to have higher power than the residual-based tests (especially noticeable for $n=50$ ). This is quite surprising, as it is exactly the opposite of what Paparoditis and Politis (2005) found for unit root tests.

\section{INSERT TABLE 4 ABOUT HERE}

Concluding, we see that the bootstrap tests all offer significant size improvements over the asymptotic test, while retaining quite good power properties. We also see that the four bootstrap tests perform quite alike, with a small advantage for the difference-based tests, both in terms of size and power. The tests based the conditional-marginal representation perform almost the same as their counterparts based on the vector representation, thus giving no reason to prefer the conditional representation over the more straightforward vector representation.

\section{Deterministic components}

In this section we will discuss how to include deterministic components in the tests. Deterministic components have to be included both in the test regression $\left(D_{t}\right.$ and $D_{t}^{r}$ in equation (6) and their bootstrap counterparts in equation (11)) and in Step 1 of the bootstrap procedure $\left(D_{t}^{s}\right.$ in equation (8)). We include deterministic components in the same way as Boswijk (1994), and consider the same five options.

The first option is to simply leave out all deterministic components, which is the case we analyzed before in the paper. Obviously this is only valid if both $\mu$ and $\tau$ in equation (1) are equal to zero.

The second and third option (Boswijk's $\xi_{\mu}^{*}$ and $\xi_{\mu}$ ) arise if there is no drift in $z_{t}(\tau=0)$. In this case we include an intercept in regression (6) and its bootstrap equivalent (11). We may either let the constant term be restricted such that it is equal to zero under the null of 
no cointegration, or let it be unrestricted. In the first case we set $D_{t}=0$ and $D_{t}^{r}=1$, in the second case we set $D_{t}=1$ and $D_{t}^{r}=0$. As in both cases $z_{t}$ does not have a drift, we do not have to include any deterministic components in Step 1 of the bootstrap procedure, hence we set $D_{t}^{s}=0$.

If the variables are generated by a process with drift, we have to include a linear trend as well as an intercept in equations (6) and (11) (Boswijk's $\xi_{\tau}^{*}$ and $\xi_{\tau}$ ). Again we can either restrict the trend to be equal to zero under the null, in which case $D_{t}=1$ and $D_{t}^{r}=t$, or we leave it unrestricted, in which case $D_{t}=(1, t)^{\prime}$ and $D_{t}^{r}=0$. As $\Delta z_{t}$ now has a nonzero mean, we include a constant term in equation (8) in Step 1 of the bootstrap procedure, i.e. we set $D_{t}^{s}=1$ in both cases.

Remark 8. While it is possible to account for the presence of deterministic components in Step 3 of the bootstrap algorithm as well, it is not necessary. By specifying the tests as we do above, the tests are similar, i.e. their asymptotic distributions do not depend on the true value of the deterministic components. Therefore building the bootstrap process with or without deterministic components will both lead to the correct limiting distribution, as long as the deterministic specification in the bootstrap test regression (11) is the same as the specification in the original regression (6), i.e. $D_{t}^{*}=D_{t}$ and $D_{t}^{r *}=D_{t}^{r}$.

Remark 9. One might want to use the test with the unrestricted constant term to deal with the situation where the variables have a drift, but the drift does not lead to a time trend in the cointegrating relation $\left(\beta^{\prime} \tau=0\right)$. However, Boswijk (1994) stresses that in this case the asymptotic distribution of the test will not be similar; it will depend on whether the drift is actually present or not. Therefore we do not consider this to be a viable option.

Remark 10. One can also adapt the bootstrap procedure mentioned in Remark 2 to the inclusion of deterministic components. As estimation in Step 1 is done under the alternative, the inclusion of deterministic components is slightly different. If we only include a constant term in the regression, we must include a constant term in equation (13) as well, hence we take $D_{t}^{s, a}=1$. If the variables are generated by a drift, and a trend is added to the regression, we let $D_{t}^{s, a}=(1, t)^{\prime}$.

To illustrate the tests with deterministic components, we perform a small simulation study. The DGP used for the simulations corresponds to the DGP used in Section 4, except that we now add deterministic components to the triangular system as follows.

$$
\begin{aligned}
y_{t} & =\mu_{1}+\tau_{1} t+\gamma x_{t}+u_{t}, \\
u_{t} & =\rho u_{t-1}+v_{1 t}, \\
\Delta\left(x_{t}-\mu_{2}-\tau_{2} t\right) & =v_{2 t} .
\end{aligned}
$$

Note that $\mu_{1}$ and $\tau_{1}$ correspond to $\beta^{\prime} \mu$ and $\beta^{\prime} \tau$ respectively in equation (4). To keep the size of the experiment down we only report simulations for $n=50$, and only for $c=0$ and 
$c=-10$. Also, we only take three combinations of $\Phi$ and $\Theta: \Phi=\Theta=0 ; \Phi=\left[\begin{array}{ll}0.2 & 0.5 \\ 0.5 & 0.2\end{array}\right]$ and $\Theta=0$; and $\Phi=0$ and $\Theta=\left[\begin{array}{ll}0.2 & 0.5 \\ 0.5 & 0.2\end{array}\right]$. We restrict our attention to the two bootstrap variants $T_{v, n}^{*}$ and $T_{v, a}^{*}$ and the asymptotic test $T_{a s}$.

We consider two models without a drift, and two where a drift is present. For the models without drift, a DGP with no deterministic components and one with just a constant term are chosen. For the models with drift, we select one DGP where the drift is canceled out in the direction of the cointegrating vector (i.e. $\tau_{1}=0$ ), and one where it is not. For each model we perform the tests with every deterministic specification that is appropriate for that specific model. The specific values used and the corresponding empirical rejection frequencies can be found in Table 5 .

\section{InsERT TABLE 5 ABOUT HERE}

It can be seen from the table that the size of the bootstrap test is satisfactory for all settings considered. As in Section 4, the null-based test has somewhat better size than the alternative-based test if there is serial correlation. The asymptotic test again has large size distortions almost everywhere. In terms of power we can draw the same conclusions as in Section 4 as well. It can also be seen that, both in terms of size and power, the rejection

frequencies for a particular deterministic specification of the tests $\left(D_{t}^{(r)}\right)$, are comparable across different specifications for the trends in the DGP ( $\mu$ and $\tau)$, thus confirming that the tests are similar.

Noticeable is that the bootstrap tests lose power if deterministic components are included unnecessarily. This is very much a small sample effect, unreported simulations for $n=100$ show this effect, although still present, is less pronounced there. The asymptotic test does not seem to lose as much power, however this can be explained by the fact that (contrary to the bootstrap tests) the size distortions of the asymptotic test increase when deterministic components are added unnecessarily. It also seems that the tests with unrestricted deterministic components are slightly more powerful than their restricted counterparts.

\section{Conclusion}

In this paper we have presented a bootstrap version of the Wald test for cointegration in a conditional single-equation ECM originally proposed by Boswijk (1994) and also considered by Pesavento (2004). We have used a multivariate sieve bootstrap method to deal with dependence in the data, and shown the asymptotic validity of the introduced test. We have also mentioned several alternative bootstrap tests, for which the asymptotic validity can be established in much the same way as we have done for our test, and demonstrated how deterministic components can be included in the test.

We have analyzed the small sample properties of our test by simulation, and compared them to those of the asymptotic test and several alternative bootstrap tests. All bootstrap 
tests clearly outperform the asymptotic test in terms of size, while retaining good power. Our bootstrap test based on the null hypothesis performs slightly better in terms of size and power than the bootstrap test based on the alternative, while the performance of the tests based on the vector representation is very similar as that of the tests based on the conditional representation. The bootstrap tests with deterministic components retain excellent size properties and are insensitive to the true value of the trends in the model as long as sufficient deterministic components are included.

It is clear from the results in our paper that our bootstrap version of the Wald ECM test is worth being considered in empirical research, as our test can be seen to improve upon the original Wald test considered by Boswijk (1994) and Pesavento (2004). The Wald ECM test easily allows for other bootstrap variants as well, such as those considered in the simulation study, or block bootstrap methods, which allow for somewhat more general DGPs. Such tests could easily be placed in the framework presented here. 


\section{A Proofs}

All bootstrap weak convergence results that we present in the following are in probability. In order to avoid too elaborate notation, we do not add this explicitly to every result and confine ourselves to stating this here instead.

In order to prove Theorem 1, we first need the following lemma.

Lemma 2. Under Assumptions 2 and 4 we have for some $a>2$

$$
\mathrm{E}^{*}\left|\varepsilon_{t}^{*}\right|^{a}=O_{p}(1)
$$

Proof of Lemma 2. Our proof follows Park (2002, Proof of Lemma 3.2). Note that ${ }^{6}$

$$
\begin{aligned}
\mathrm{E}^{*}\left|\varepsilon_{t}^{*}\right|^{a} & =\frac{1}{n} \sum_{t=1}^{n}\left|\hat{\varepsilon}_{q, t}-\frac{1}{n} \sum_{t=1}^{n} \hat{\varepsilon}_{q, t}\right|^{a} \\
& =\frac{1}{n} \sum_{t=1}^{n}\left|\hat{\varepsilon}_{q, t}-\varepsilon_{q, t}+\varepsilon_{q, t}-\varepsilon_{t}+\varepsilon_{t}-\frac{1}{n} \sum_{t=1}^{n} \hat{\varepsilon}_{q, t}\right|^{a} \\
& \leq 4^{a-1} \frac{1}{n} \sum_{t=1}^{n}\left\{\left|\hat{\varepsilon}_{q, t}-\varepsilon_{q, t}\right|^{a}+\left|\varepsilon_{q, t}-\varepsilon_{t}\right|^{a}+\left|\varepsilon_{t}\right|^{a}+\left|\frac{1}{n} \sum_{t=1}^{n} \hat{\varepsilon}_{q, t}\right|^{a}\right\} \\
& =c\left(A_{n}+B_{n}+C_{n}+D_{n}\right)
\end{aligned}
$$

where

$$
\begin{aligned}
A_{n} & =\frac{1}{n} \sum_{t=1}^{n}\left|\varepsilon_{t}\right|^{a} & B_{n} & =\frac{1}{n} \sum_{t=1}^{n}\left|\varepsilon_{q, t}-\varepsilon_{t}\right|^{a} \\
C_{n} & =\frac{1}{n} \sum_{t=1}^{n}\left|\hat{\varepsilon}_{q, t}-\varepsilon_{q, t}\right|^{a} & D_{n} & =\left|\frac{1}{n} \sum_{t=1}^{n} \hat{\varepsilon}_{q, t}\right|^{a}
\end{aligned}
$$

and $c=4^{a-1}$ is a constant not depending on $n$. Note that $\varepsilon_{q, t}$ is defined as

$$
\varepsilon_{q, t}=u_{t}-\sum_{j=1}^{q} \Phi_{j} u_{t-j}=\varepsilon_{t}+\sum_{j=q+1}^{\infty} \Phi_{j} u_{t-j} .
$$

We first look at $A_{n}$. By the weak law of large numbers, $\frac{1}{n} \sum_{t=1}^{n}\left|\varepsilon_{t}\right|^{a} \stackrel{p}{\rightarrow} \mathrm{E}\left|\varepsilon_{t}\right|^{a}$. As by Assumption $2 \mathrm{E}\left|\varepsilon_{t}\right|^{a}=O(1)$, we have that $A_{n}=O_{p}(1)$.

\footnotetext{
${ }^{6}$ Every convex function $f(x)$ has the property that $f\left(\sum_{i=1}^{k} x_{i} / k\right) \leq \sum_{i=1}^{k} f\left(x_{i}\right) / k$. Applying this to the function $f(x)=|x|^{a}$, we have$$
\left|\sum_{i=1}^{k} x_{i}\right|^{a}=k^{a}\left|\sum_{i=1}^{k} x_{i} / k\right|^{a} \leq k^{a} \sum_{i=1}^{k}\left|x_{i}\right|^{a} / k=k^{a-1} \sum_{i=1}^{k}\left|x_{i}\right|^{a} .
$$ 
For $B_{n}$, we wish to show that $\mathrm{E}\left|\varepsilon_{q, t}-\varepsilon_{t}\right|^{a}=o\left(q^{-a}\right)$ as $\frac{1}{n} \sum_{t=1}^{n}\left|\varepsilon_{q, t}-\varepsilon_{t}\right|^{a} \stackrel{p}{\rightarrow} \mathrm{E}\left|\varepsilon_{q, t}-\varepsilon_{t}\right|^{a}$. Using Minkowski's inequality we have

$$
\begin{aligned}
\mathrm{E}\left|\varepsilon_{q, t}-\varepsilon_{t}\right|^{a} & =\mathrm{E}\left|\sum_{j=q+1}^{\infty} \Phi_{j} u_{t-j}\right|^{a} \leq\left(\sum_{j=q+1}^{\infty}\left(\mathrm{E}\left|\Phi_{j} u_{t-j}\right|^{a}\right)^{1 / a}\right)^{a} \\
& \leq\left(\sum_{j=q+1}^{\infty}\left|\Phi_{j}\right|\left(\mathrm{E}\left|u_{t-j}\right|^{a}\right)^{1 / a}\right)^{a}=\left(\left(\mathrm{E}\left|u_{t}\right|^{a}\right)^{1 / a} \sum_{j=q+1}^{\infty}\left|\Phi_{j}\right|\right)^{a} \\
& =\mathrm{E}\left|u_{t}\right|^{a}\left(\sum_{j=q+1}^{\infty}\left|\Phi_{j}\right|\right)^{a}=o\left(q^{-a}\right)
\end{aligned}
$$

The final step comes from Hannan and Kavalieris (1986), who show that if $\sum_{j=0}^{\infty} j\left|\Psi_{j}\right|<\infty$, then $\sum_{j=0}^{\infty} j\left|\Phi_{j}\right|<\infty\left(\right.$ where $\left.\Phi(L)=\Psi(L)^{-1}\right)$. They also show that if $\sum_{j=0}^{\infty} j\left|\Phi_{j}\right|<\infty$, then $\sum_{j=q+1}^{\infty}\left|\Phi_{j}\right|=o\left(q^{-1}\right)$.

Next we turn to $C_{n}$. We can write

$$
\begin{aligned}
\hat{\varepsilon}_{q, t} & =u_{t}-\sum_{j=1}^{q} \hat{\Phi}_{j} u_{t-j}=\varepsilon_{q, t}+\sum_{j=1}^{q} \Phi_{j} u_{t-j}-\sum_{j=1}^{q} \hat{\Phi}_{j} u_{t-j} \\
& =\varepsilon_{q, t}-\sum_{j=1}^{q}\left(\hat{\Phi}_{j}-\Phi_{q, j}\right) u_{t-j}-\sum_{j=1}^{q}\left(\Phi_{q, j}-\Phi_{j}\right) u_{t-j}
\end{aligned}
$$

where $\Phi_{q, j}$ is defined as the coefficient of $y_{t-j}$ in the best linear predictor of $y_{t}$ in terms of $y_{t-1}, \ldots, y_{t-q}$. Then

$$
\left|\hat{\varepsilon}_{q, t}-\varepsilon_{q, t}\right|^{a} \leq 2^{a-1}\left(\left|\sum_{j=1}^{q}\left(\hat{\Phi}_{j}-\Phi_{q, j}\right) u_{t-j}\right|^{a}+\left|\sum_{j=1}^{q}\left(\Phi_{q, j}-\Phi_{j}\right) u_{t-j}\right|^{a}\right) .
$$

We define

$$
C_{1 n}=\frac{1}{n} \sum_{t=1}^{n}\left|\sum_{j=1}^{q}\left(\hat{\Phi}_{j}-\Phi_{q, j}\right) u_{t-j}\right|^{a}, \quad C_{2 n}=\frac{1}{n} \sum_{t=1}^{n}\left|\sum_{j=1}^{q}\left(\Phi_{q, j}-\Phi_{j}\right) u_{t-j}\right|^{a}
$$

and show that $C_{1 n}, C_{2 n}=o_{p}(1)$. Then we have that

$$
\begin{aligned}
C_{1 n} & =\frac{1}{n} \sum_{t=1}^{n}\left|\sum_{j=1}^{q}\left(\hat{\Phi}_{j}-\Phi_{q, j}\right) u_{t-j}\right|^{a} \leq q^{a-1} \frac{1}{n} \sum_{t=1}^{n} \sum_{j=1}^{q}\left|\left(\hat{\Phi}_{j}-\Phi_{q, j}\right)\right|^{a}\left|u_{t-j}\right|^{a} \\
& \leq q^{a-1}\left(\max _{1 \leq j \leq q}\left|\hat{\Phi}_{j}-\Phi_{q, j}\right|^{a}\right) \frac{1}{n} \sum_{t=1}^{n} \sum_{j=1}^{q}\left|u_{t-j}\right|^{a}
\end{aligned}
$$


As every value of $\left|u_{t-j}\right|^{a}$ for $j=1-q, \ldots, n-1$ occurs at most $q$ times in the double sum $\sum_{t=1}^{n} \sum_{j=1}^{q}\left|u_{t-j}\right|^{a}$, we have that

$$
\begin{aligned}
C_{1 n} & \leq q^{a-1}\left(\max _{1 \leq j \leq q}\left|\hat{\Phi}_{j}-\Phi_{q, j}\right|^{a}\right) \frac{1}{n} \sum_{t=1}^{n} \sum_{j=1}^{q}\left|u_{t-j}\right|^{a} \\
& \leq q^{a}\left(\max _{1 \leq j \leq q}\left|\hat{\Phi}_{j}-\Phi_{q, j}\right|^{a}\right) \frac{1}{n}\left(\sum_{t=0}^{n-1}\left|u_{t}\right|^{a}+\sum_{t=-1}^{1-q}\left|u_{t}\right|^{a}\right) \\
& =O_{p}\left((\ln n / n)^{a / 2}\right)\left(q^{a} / n\right) O_{p}(n)=O_{p}\left(q^{a}(\ln n / n)^{a / 2}\right),
\end{aligned}
$$

where we use that

$$
\max _{1 \leq j \leq q}\left|\hat{\Phi}_{j}-\Phi_{q, j}\right|=O_{p}\left((\ln n / n)^{1 / 2}\right)
$$

from Hannan and Kavalieris (1986). As $q=o\left((n / \ln n)^{1 / 2}\right), C_{1 n}=o_{p}(1)$.

For $C_{2 n}$, we have that $C_{2 n} \stackrel{p}{\rightarrow} \mathrm{E}\left|\sum_{j=1}^{q}\left(\Phi_{q, j}-\Phi_{j}\right) u_{t-j}\right|^{a}$. Then, again using Minkowski's inequality, we have

$$
\begin{aligned}
\mathrm{E}\left|\sum_{j=1}^{q}\left(\Phi_{q, j}-\Phi_{j}\right) u_{t-j}\right|^{a} & \leq\left(\sum_{j=1}^{q}\left(\mathrm{E}\left|\left(\Phi_{q, j}-\Phi_{j}\right) u_{t-j}\right|^{a}\right)^{1 / a}\right)^{a} \\
& \leq\left(\left(\mathrm{E}\left|u_{t}\right|^{a}\right)^{1 / a} \sum_{j=1}^{q}\left|\Phi_{q, j}-\Phi_{j}\right|\right)^{a} \\
& =\mathrm{E}\left|u_{t}\right|^{a}\left(\sum_{j=1}^{q}\left|\Phi_{q, j}-\Phi_{j}\right|\right)^{a}
\end{aligned}
$$

Again from Hannan and Kavalieris (1986) and Bühlmann (1995), we have that

$$
\sum_{j=1}^{q}\left|\Phi_{q, j}-\Phi_{j}\right| \leq c \sum_{j=q+1}^{\infty}\left|\Phi_{j}\right|=o\left(q^{-1}\right)
$$

with $c$ some constant. Hence, $C_{2 n}=o\left(q^{-a}\right)$ which completes the proof for $C_{n}$.

Finally, we look at $D_{n}$. We want to show that

$$
\frac{1}{n} \sum_{t=1}^{n} \hat{\varepsilon}_{q, t}=\frac{1}{n} \sum_{t=1}^{n} \varepsilon_{q, t}+o_{p}(1)=\frac{1}{n} \sum_{t=1}^{n} \varepsilon_{t}+o_{p}(1) .
$$

Using equations (17) and (18) we can write

$$
\hat{\varepsilon}_{q, t}=\varepsilon_{t}+\sum_{j=q+1}^{\infty} \Phi_{j} u_{t-j}-\sum_{j=1}^{q}\left(\hat{\Phi}_{j}-\Phi_{q, j}\right) u_{t-j}-\sum_{j=1}^{q}\left(\Phi_{q, j}-\Phi_{j}\right) u_{t-j} .
$$


Hence, what we need to show is that

$$
\begin{array}{r}
\frac{1}{n} \sum_{t=1}^{n} \sum_{j=q+1}^{\infty} \Phi_{j} u_{t-j} \stackrel{p}{\rightarrow} 0 \\
\frac{1}{n} \sum_{t=1}^{n} \sum_{j=1}^{q}\left(\Phi_{q, j}-\Phi_{j}\right) u_{t-j} \stackrel{p}{\rightarrow} 0 \\
\frac{1}{n} \sum_{t=1}^{n} \sum_{j=1}^{q}\left(\hat{\Phi}_{j}-\Phi_{q, j}\right) u_{t-j} \stackrel{p}{\rightarrow} 0 .
\end{array}
$$

First define

$$
S_{n}(i, j)=\sum_{t=1}^{n} \varepsilon_{t-i-j} \quad \text { and } T_{n}(i)=\sum_{t=1}^{n} u_{t-i} .
$$

As $u_{t}=\sum_{j=0}^{\infty} \Psi_{j} \varepsilon_{t-j}$, it follows that

$$
T_{n}(i)=\sum_{t=1}^{n} \sum_{j=0}^{\infty} \Psi_{j} \varepsilon_{t-i-j}=\sum_{j=0}^{\infty} \Psi_{j} S_{n}(i, j) .
$$

Note that the $k$-th element of $S_{n}, S_{k, n}(i, j)$ is a univariate martingale.

By successive applications of Doob's inequality and Burkholder's inequality, we have that

$$
\begin{aligned}
\mathrm{E}\left(\max _{1 \leq m \leq n}\left|S_{m}(i, j)\right|^{a}\right) & =\mathrm{E}\left(\max _{1 \leq m \leq n}\left(\sum_{k=1}^{1+l} S_{k, m}^{2}(i, j)\right)^{a / 2}\right) \\
& \leq(1+l)^{a / 2-1} \sum_{k=1}^{1+l} \mathrm{E}\left(\max _{1 \leq m \leq n}\left|S_{k, m}(i, j)\right|^{a}\right) \\
& \leq(1+l)^{a / 2-1}\left(\frac{a}{a-1}\right)^{a} \sum_{k=1}^{1+l} \mathrm{E}\left|S_{k, n}(i, j)\right|^{a} \\
& \leq(1+l)^{a / 2-1}\left(\frac{a}{a-1}\right)^{a} C_{a / 2} \sum_{k=1}^{1+l} \mathrm{E}\left(\sum_{t=1}^{n} \varepsilon_{k, t-i-j}^{2}\right)^{a / 2} \\
& \leq(1+l)^{a / 2-1}\left(\frac{a}{a-1}\right)^{a} C_{a / 2} n^{a / 2-1} \sum_{k=1}^{1+l} \sum_{t=1}^{n} \mathrm{E}\left|\varepsilon_{k, t-i-j}\right|^{a} .
\end{aligned}
$$

As $l$ is finite, and $\mathrm{E}\left|\varepsilon_{k, t}\right|^{a}=O(1)$ we can find a constant $c_{S}$ such that

$$
\mathrm{E}\left(\max _{1 \leq m \leq n}\left|S_{m}(i, j)\right|^{a}\right) \leq c_{S} n^{a / 2}
$$


Furthermore, because

$$
\begin{aligned}
{\left[\mathrm{E}\left(\max _{1 \leq m \leq n}\left|T_{m}(i)\right|^{a}\right)\right]^{1 / a} } & =\left[\mathrm{E}\left(\max _{1 \leq m \leq n}\left|\sum_{j=0}^{\infty} \Psi_{j} S_{m}(i, j)\right|^{a}\right)\right]^{1 / a} \\
& \leq \sum_{j=0}^{\infty}\left[\mathrm{E}\left(\max _{1 \leq m \leq n}\left|\Psi_{j} S_{m}(i, j)\right|\right)^{a}\right]^{1 / a} \\
& \leq \sum_{j=0}^{\infty}\left|\Psi_{j}\right|\left[\mathrm{E}\left(\max _{1 \leq m \leq n}\left|S_{m}(i, j)\right|\right)^{a}\right]^{1 / a}
\end{aligned}
$$

we have

$$
\mathrm{E}\left(\max _{1 \leq m \leq n}\left|T_{m}(i)\right|^{a}\right) \leq c_{T} n^{a / 2}
$$

where $c_{T}$ is a constant not depending on $n$.

Now define

$$
L_{n}=\sum_{t=1}^{n} \sum_{j=q+1}^{\infty} \Phi_{j} u_{t-j}=\sum_{j=q+1}^{\infty} \Phi_{j} \sum_{t=1}^{n} u_{t-j}=\sum_{j=q+1}^{\infty} \Phi_{j} T_{n}(j) .
$$

Then we have (again using Minkowski's inequality)

$$
\begin{aligned}
{\left[\mathrm{E}\left(\max _{1 \leq m \leq n}\left|L_{m}(i)\right|^{a}\right)\right]^{1 / a} } & =\left[\mathrm{E}\left(\max _{1 \leq m \leq n}\left|\sum_{j=q+1}^{\infty} \Phi_{j} T_{m}(j)\right|^{a}\right)\right]^{1 / a} \\
& \leq \sum_{j=q+1}^{\infty}\left[\mathrm{E}\left(\max _{1 \leq m \leq n}\left|\Phi_{j} T_{m}(j)\right|\right)^{a}\right]^{1 / a} \\
& \leq \sum_{j=q+1}^{\infty}\left|\Phi_{j}\right|\left[\mathrm{E}\left(\max _{1 \leq m \leq n}\left|T_{m}(j)\right|\right)^{a}\right]^{1 / a} \\
& \leq c_{L} q^{-1} n^{1 / 2}
\end{aligned}
$$

and hence

$$
\mathrm{E}\left(\max _{1 \leq m \leq n}\left|L_{m}(i)\right|^{a}\right) \leq c q^{-a} n^{a / 2} .
$$

Then for the $k$-th element of $L_{m}$, we have that $\mathrm{E}\left(\max _{1 \leq m \leq n}\left|L_{k, m}(i)\right|^{a}\right) \leq c_{k} q^{-a} n^{a / 2}$. We can then refer to Móricz (1976, pp. 309-310, below (4.8)), from where we can deduce that if the above holds, then for any $\delta>0$

$$
L_{k, n}=o_{p}\left(q^{-1} n^{1 / 2}(\ln n)^{1 / a}(\ln \ln n)^{(1+\delta) / a}\right)=o_{p}(n)
$$


and hence

$$
L_{n}=o_{p}\left(q^{-1} n^{1 / 2}(\ln n)^{1 / a}(\ln \ln n)^{(1+\delta) / a}\right)=o_{p}(n),
$$

which proves (21). To establish (22) we let

$$
M_{n}=\sum_{j=1}^{q}\left(\Phi_{q, j}-\Phi_{j}\right) \sum_{t=1}^{n} u_{t-j}=\sum_{j=1}^{q}\left(\Phi_{q, j}-\Phi_{j}\right) T_{n}(j)
$$

We can then in exactly the same way as before derive that

$$
\left[\mathrm{E}\left(\max _{1 \leq m \leq n}\left|M_{m}\right|^{a}\right)\right]^{1 / a} \leq \sum_{j=1}^{q}\left|\Phi_{q, j}-\Phi_{j}\right|\left[\mathrm{E}\left(\max _{1 \leq m \leq n}\left|T_{m}(j)\right|^{a}\right)\right]^{1 / a} \leq c_{M} q^{-1} n^{1 / 2}
$$

and hence

$$
M_{n}=o_{p}\left(q^{-1} n^{1 / 2}(\ln n)^{1 / a}(\ln \ln n)^{(1+\delta) / a}\right)=o_{p}(n) .
$$

Finally, let

$$
N_{n}=\sum_{j=1}^{q}\left(\hat{\Phi}_{j}-\Phi_{q, j}\right) \sum_{t=1}^{n} u_{t-j}=\sum_{j=1}^{q}\left(\hat{\Phi}_{j}-\Phi_{q, j}\right) T_{n}(j)
$$

and derive

$$
\begin{aligned}
\left|N_{n}\right| & =\left|\sum_{j=1}^{q}\left(\hat{\Phi}_{j}-\Phi_{q, j}\right) \sum_{t=1}^{n} u_{t-j}\right| \leq \sum_{j=1}^{q}\left|\left(\hat{\Phi}_{j}-\Phi_{q, j}\right) \sum_{t=1}^{n} u_{t-j}\right| \\
& \leq \sum_{j=1}^{q}\left|\hat{\Phi}_{j}-\Phi_{q, j}\right|\left|\sum_{t=1}^{n} u_{t-j}\right| \leq\left(\max _{1 \leq j \leq q}\left|\hat{\Phi}_{j}-\Phi_{q, j}\right|\right) Q_{n},
\end{aligned}
$$

where

$$
Q_{n}=\sum_{j=1}^{q}\left|\sum_{t=1}^{n} u_{t-j}\right|=\sum_{j=1}^{q}\left|T_{n}(j)\right|
$$

Then

$$
\left[\mathrm{E}\left(\max _{1 \leq m \leq n}\left|Q_{m}\right|^{a}\right)\right]^{1 / a} \leq \sum_{j=1}^{q}\left[\mathrm{E}\left(\max _{1 \leq m \leq n}\left|T_{m}(j)\right|^{a}\right)\right]^{1 / a} \leq c_{Q} q^{-1} n^{1 / 2}
$$


such that

$$
Q_{n}=o_{p}\left(q n^{1 / 2}(\ln n)^{1 / a}(\ln \ln n)^{(1+\delta) / a}\right)
$$

Then by (19) we have that

$$
N_{n}=O_{p}\left((\ln n / n)^{a}\right) o_{p}\left(q n^{1 / 2}(\ln n)^{1 / a}(\ln \ln n)^{(1+\delta) / a}\right)=o_{p}(n)
$$

which shows that (23) holds. This shows that $D_{n}=O_{p}(1)$, and the proof is complete.

Before proceeding with the proof of Theorem 1, we need one additional lemma to ensure that the covariance matrix of the bootstrap errors correctly mimics that of the original errors.

Lemma 3. Under Assumptions 2 and 4 we have that

$$
\Sigma^{*}=\mathrm{E}^{*}\left(\varepsilon_{t}^{*} \varepsilon_{t}^{* \prime}\right)=\Sigma+o_{p}(1)
$$

Proof of Lemma 3. This proof follows Paparoditis (1996, Proof of Theorem 2.5, p. 288). First note that $\mathrm{E}^{*}\left(\varepsilon_{t}^{*} \varepsilon_{t}^{* \prime}\right)=n^{-1} \sum_{t=1}^{n} \tilde{\varepsilon}_{q, t} \tilde{\varepsilon}_{q, t}^{\prime}$. Then

$$
\begin{aligned}
\left|\Sigma^{*}-\Sigma\right| & =\left|n^{-1} \sum_{t=1}^{n} \tilde{\varepsilon}_{q, t} \tilde{\varepsilon}_{q, t}^{\prime}-\Sigma\right|=\left|n^{-1} \sum_{t=1}^{n}\left(\tilde{\varepsilon}_{q, t} \tilde{\varepsilon}_{q, t}^{\prime}-\varepsilon_{t} \varepsilon_{t}^{\prime}\right)\right|+\left|n^{-1} \sum_{t=1}^{n} \varepsilon_{t} \varepsilon_{t}^{\prime}-\Sigma\right| \\
& =n^{-1} \sum_{t=1}^{n}\left|\tilde{\varepsilon}_{q, t} \tilde{\varepsilon}_{q, t}^{\prime}-\varepsilon_{t} \varepsilon_{t}^{\prime}\right|+o_{p}(1) \\
& =n^{-1} \sum_{t=1}^{n}\left|\left(\tilde{\varepsilon}_{q, t}-\varepsilon_{t}\right) \tilde{\varepsilon}_{q, t}^{\prime}+\varepsilon_{t}\left(\tilde{\varepsilon}_{q, t}-\varepsilon_{t}\right)^{\prime}\right|+o_{p}(1) \\
& \leq n^{-1} \sum_{t=1}^{n}\left|\tilde{\varepsilon}_{q, t}-\varepsilon_{t}\right|\left|\tilde{\varepsilon}_{q, t}\right|+n^{-1} \sum_{t=1}^{n}\left|\varepsilon_{t}\right|\left|\tilde{\varepsilon}_{q, t}-\varepsilon_{t}\right|+o_{p}(1) \\
& \leq \max _{1 \leq t \leq n}\left|\tilde{\varepsilon}_{q, t}\right| n^{-1} \sum_{t=1}^{n}\left|\tilde{\varepsilon}_{q, t}-\varepsilon_{t}\right|+\max _{1 \leq t \leq n}\left|\varepsilon_{t}\right| n^{-1} \sum_{t=1}^{n}\left|\tilde{\varepsilon}_{q, t}-\varepsilon_{t}\right|+o_{p}(1),
\end{aligned}
$$

as $n^{-1} \sum_{t=1}^{n} \varepsilon_{t} \varepsilon_{t}^{\prime}-\Sigma \stackrel{p}{\rightarrow} 0$ by the weak law of large numbers.

Note that

$$
\left|\tilde{\varepsilon}_{q, t}\right| \leq\left|\hat{\varepsilon}_{q, t}\right|+\left|n^{-1} \sum_{t=1}^{n} \hat{\varepsilon}_{t}\right| \leq\left|\hat{\varepsilon}_{q, t}-\varepsilon_{q, t}\right|+\left|\varepsilon_{q, t}-\varepsilon_{t}\right|+\left|\varepsilon_{t}\right|+\left|n^{-1} \sum_{t=1}^{n} \hat{\varepsilon}_{q, t}\right|
$$

and

$$
\left|\tilde{\varepsilon}_{q, t}-\varepsilon_{t}\right|=\left|\hat{\varepsilon}_{q, t}-n^{-1} \sum_{t=1}^{n} \hat{\varepsilon}_{q, t}-\varepsilon_{t}\right| \leq\left|\hat{\varepsilon}_{t q},-\varepsilon_{q, t}\right|+\left|\varepsilon_{q, t}-\varepsilon_{t}\right|+\left|n^{-1} \sum_{t=1}^{n} \hat{\varepsilon}_{t}\right| .
$$


It now follows that $\left|\Sigma^{*}-\Sigma\right|=o_{p}(1)$ by applying the methods from the proof of Lemma 2 (with $a=1$ ).

Proof of Theorem 1. In this proof we draw heavily on results by Einmahl (1987), as in Chang et al. (2006). Therefore, we first need to introduce notation used by Einmahl (1987). Let $\left(\mathbb{R}^{d},|\cdot|\right)$ denote the $d$-dimensional Euclidean space. Let $C_{d}[0,1]$ be the space of all continuous $\mathbb{R}^{d}$-valued functions on $[0,1]$ endowed with the sup-norm $\|\cdot\|$.

Let $\lambda\left(Q_{1}, Q_{2}, \delta\right)$ denote the $\delta$-distance of two measures $Q_{1}$ and $Q_{2}$, that is

$$
\lambda\left(Q_{1}, Q_{2}, \delta\right)=\sup \left\{Q_{1}(A)-Q_{2}\left(A^{\delta}\right): A \subseteq C_{d}[0,1] \text { closed }\right\}
$$

where $A^{\delta}=\left\{g \in C_{d}[0,1]: \exists f \in A,\|f-g\|<\delta\right\}$. Then Einmahl (1987) shows that

$$
\lambda\left(W_{n}^{*}, W, \delta\right) \leq c \delta^{-a} K_{a n}^{*},
$$

holds for all $\delta$ if $2<a<4$, and $\delta>K_{a n}^{* \gamma}$ if $a \geq 4$; where $K_{a n}^{*}=\sum_{t=1}^{n} \mathrm{E}^{*}\left|\varepsilon_{t}^{*}\right|^{a}, c$ is a positive constant depending only on $a, l$ and $\gamma$, and $0<\gamma<1 /(2 a-4)$.

By noting that $K_{a n}^{*}=\sum_{t=1}^{n} \mathrm{E}^{*}\left|\varepsilon_{t}^{*}\right|^{a}=n \mathrm{E}^{*}\left|\varepsilon_{t}^{*}\right|^{a}$, we can, as in Chang et al. (2006), transform this into the following condition:

$$
\mathrm{P}^{*}\left\{\sup _{0 \leq r \leq 1}\left|W_{n}^{*}(r)-W(r)\right|>n^{-1 / 2} c_{n}\right\} \leq K n c_{n}^{-a} \mathrm{E}^{*}\left|\varepsilon_{t}^{*}\right|^{a}
$$

for any sequence $\left\{c_{n}\right\}, c_{n}=n^{1 / a+\delta_{2}}$ for any $\delta_{2}>0$, where $K$ is an absolute constant depending only on $a$ and $l$.

Once we have the result in (25), we can take $0<\delta_{2}<1 / 2-1 / a$, or alternatively, $\delta_{2}=1 / 2-1 / a-\epsilon$, where $0<\epsilon(<1 / 2-1 / a)$. Then on the left-hand side we have $n^{-1 / 2} c_{n}=$ $n^{-1 / 2+1 / a+\delta_{2}}=n^{-\epsilon}$. On the right-hand side we have $c_{n}^{-a}=\left(n^{1 / a+\delta_{2}}\right)^{-a}=n^{-1-a \delta_{2}}$, to show that

$$
\mathrm{P}^{*}\left\{\sup _{0 \leq r \leq 1}\left|W_{n}^{*}(r)-W(r)\right|>n^{-\epsilon}\right\} \leq K n^{-\left(1+a \delta_{2}\right)} \mathrm{E}^{*}\left|\varepsilon_{t}^{*}\right|^{a},
$$

from which we can deduce that, as $n \rightarrow \infty$,

$$
\sup _{0 \leq r \leq 1}\left|W_{n}^{*}(r)-W(r)\right|=o_{p}^{*}(1)
$$

Note that the bootstrap stochastic order symbols $O_{p}^{*}(\cdot)$ and $o_{p}^{*}(\cdot)$ are defined for the bootstrap sample in the same way as $O_{p}(\cdot)$ and $o_{p}(\cdot)$ are for the original sample (see Chang and Park, 2003, Remark 1). 
Proof of Theorem 2. Using the Beveridge-Nelson decomposition, we can write

$$
\begin{aligned}
\varepsilon_{t}^{*} & =u_{t}^{*}-\sum_{j=1}^{q} \hat{\Phi}_{j} u_{t-j}^{*} \\
& =\left(I-\sum_{j=1}^{q} \hat{\Phi}_{j}\right) u_{t}^{*}+\sum_{i=1}^{q} \sum_{j=i}^{q} \hat{\Phi}_{j}\left(u_{t-i+1}^{*}-u_{t-i}^{*}\right) \\
& =\hat{\Phi}(1) u_{t}^{*}-\sum_{i=1}^{q} \sum_{j=i}^{q} \hat{\Phi}_{j}\left(u_{t-i}^{*}-u_{t-i+1}^{*}\right)
\end{aligned}
$$

and hence

$$
u_{t}^{*}=\hat{\Psi}(1) \varepsilon_{t}^{*}+\hat{\Psi}(1) \sum_{i=1}^{q}\left(\sum_{j=i}^{q} \hat{\Phi}_{j}\right)\left(u_{t-i}^{*}-u_{t-i+1}^{*}\right)=\hat{\Psi}(1) \varepsilon_{t}^{*}+\left(\bar{u}_{t-1}^{*}-\bar{u}_{t}^{*}\right)
$$

where $\bar{u}_{t-1}^{*}=\hat{\Psi}(1) \sum_{i=1}^{q}\left(\sum_{j=i}^{q} \hat{\Phi}_{j}\right) u_{t-i}^{*}$ and $\hat{\Psi}(1)=\hat{\Phi}(1)^{-1}$. Then

$$
\begin{aligned}
B_{n}^{*}(r) & =n^{-1 / 2} \sum_{t=1}^{[n r]} u_{t}^{*} \\
& =n^{-1 / 2} \sum_{t=1}^{[n r]} \hat{\Psi}(1) \varepsilon_{t}^{*}+n^{-1 / 2} \sum_{t=1}^{[n r]}\left(\bar{u}_{t-1}^{*}-\bar{u}_{t}^{*}\right) \\
& =\hat{\Psi}(1) W_{n}^{*}(r)+n^{-1 / 2}\left(\bar{u}_{0}^{*}-\bar{u}_{[n r]}^{*}\right)
\end{aligned}
$$

Hence, we need to show that

$$
\begin{aligned}
& \hat{\Phi}(1) \stackrel{p}{\rightarrow} \Phi(1) \\
& \mathrm{P}^{*}\left\{\max _{1 \leq t \leq n}\left|n^{-1 / 2} \bar{u}_{t}^{*}\right|>\epsilon\right\}=o_{p}(1)
\end{aligned}
$$

We can follow Chang et al. (2006, Proof of Theorem 3.3) for the proofs of these result.

We first show (26). Using equations (19) and (20) we have that

$$
\begin{aligned}
|\hat{\Phi}(1)-\Phi(1)| & =\left|\sum_{j=1}^{q}\left(\hat{\Phi}_{j}-\Phi_{q, j}\right)+\sum_{j=1}^{q}\left(\Phi_{q, j}-\Phi_{j}\right)-\sum_{q+1}^{\infty} \Phi_{j}\right| \\
& \leq \sum_{j=1}^{q}\left|\hat{\Phi}_{j}-\Phi_{q, j}\right|+\sum_{j=1}^{q}\left|\Phi_{q, j}-\Phi_{j}\right|+\sum_{q+1}^{\infty}\left|\Phi_{j}\right| \\
& =O_{p}\left(q(\ln n / n)^{1 / 2}\right)+o\left(q^{-1}\right) .
\end{aligned}
$$


Hence,

$$
\hat{\Phi}(1)=\Phi(1)+o_{p}(1) .
$$

This proves (26).

To prove (27), we have as in Park (2002)

$$
\mathrm{P}^{*}\left\{\max _{1 \leq t \leq n}\left|n^{-1 / 2} \bar{u}_{t}^{*}\right|>\epsilon\right\} \leq n \mathrm{P}^{*}\left\{\left|n^{-1 / 2} \bar{u}_{t}^{*}\right|>\epsilon\right\} \leq\left(1 / \epsilon^{a}\right) n^{1-a / 2} \mathrm{E}^{*}\left|\bar{u}_{t}^{*}\right|^{a}
$$

The second inequality follows from Markov's inequality. Hence, we have to show that

$$
n^{1-a / 2} \mathrm{E}^{*}\left|\bar{u}_{t}^{*}\right|^{a}=o_{p}(1) .
$$

For large $n$ we can write

$$
u_{t}^{*}=\sum_{j=0}^{\infty} \hat{\Psi}_{j} \varepsilon_{t-j}^{*}
$$

and furthermore

$$
\bar{u}_{t}^{*}=\sum_{j=0}^{\infty} \bar{\Psi}_{j}^{*} \varepsilon_{t-j}^{*}
$$

where

$$
\bar{\Psi}_{j}^{*}=\sum_{i=j+1}^{\infty} \hat{\Psi}_{j} .
$$

By successive application of the Marcinkiewicz-Zygmund inequality for vectors (De Acosta, 1981; Berger, 1991) and Minkowski's inequality, we have

$$
\begin{aligned}
\mathrm{E}^{*}|| \bar{u}_{t}^{*}\left|-\mathrm{E}^{*}\right| \bar{u}_{t}||^{a} & \leq c_{a} \mathrm{E}^{*}\left(\sum_{j=0}^{\infty}\left|\bar{\Psi}_{j}^{*} \varepsilon_{t-j}^{*}\right|^{2}\right)^{a / 2} \\
& \leq c_{a}\left(\sum_{j=0}^{\infty}\left(\mathrm{E}^{*}\left|\bar{\Psi}_{j}^{*} \varepsilon_{t-j}^{*}\right|^{a}\right)^{2 / a}\right)^{a / 2} \\
& \leq c_{a}\left(\sum_{j=0}^{\infty}\left|\bar{\Psi}_{j}^{*}\right|^{2}\left(\mathrm{E}^{*}\left|\varepsilon_{t-j}^{*}\right|^{a}\right)^{2 / a}\right)^{a / 2} \\
& =c_{a}\left(\sum_{j=0}^{\infty}\left|\bar{\Psi}_{j}^{*}\right|^{2}\right)^{a / 2} \mathrm{E}^{*}\left|\varepsilon_{t}^{*}\right|^{a}
\end{aligned}
$$


for some constant $c_{a}$. Phillips and Solo (1992, p. 973) show that a sufficient condition for $\sum_{j=1}^{\infty}\left|\bar{\Psi}_{j}^{*}\right|^{2}=O_{p}(1)$ is

$$
\sum_{j=1}^{\infty} j^{1 / 2}\left|\hat{\Psi}_{j}\right|=O_{p}(1)
$$

This will in turn hold if (Hannan and Kavalieris, 1986)

$$
\sum_{j=1}^{q} j^{1 / 2}\left|\hat{\Phi}_{j}\right|=O_{p}(1)
$$

We have

$$
\begin{aligned}
\sum_{j=1}^{q} j^{1 / 2}\left|\hat{\Phi}_{j}\right| & =\sum_{j=1}^{q} j^{1 / 2}\left|\hat{\Phi}_{j}-\Phi_{q, j}+\Phi_{q, j}-\Phi_{j}+\Phi_{j}\right| \\
& \leq \sum_{j=1}^{q} j^{1 / 2}\left|\hat{\Phi}_{j}-\Phi_{q, j}\right|+\sum_{j=1}^{q} j^{1 / 2}\left|\Phi_{q, j}-\Phi_{j}\right|+\sum_{j=1}^{q} j^{1 / 2}\left|\Phi_{j}\right| \\
& \leq q^{1 / 2} \sum_{j=1}^{q}\left|\hat{\Phi}_{j}-\Phi_{q, j}\right|+q^{1 / 2} \sum_{j=1}^{q}\left|\Phi_{q, j}-\Phi_{j}\right|+\sum_{j=1}^{\infty} j^{1 / 2}\left|\Phi_{j}\right| \\
& \leq q^{3 / 2} \max _{1 \leq j \leq q}\left|\hat{\Phi}_{j}-\Phi_{q, j}\right|+q^{1 / 2} \sum_{j=1}^{q}\left|\Phi_{q, j}-\Phi_{j}\right|+\sum_{j=1}^{\infty} j^{1 / 2}\left|\Phi_{j}\right| \\
& =O_{p}\left(q^{3 / 2}(\ln n / n)^{1 / 2}\right)+o_{p}\left(q^{-1}\right)+O(1)=O_{p}(1)
\end{aligned}
$$

by (19), (20) and Assumption $4^{\prime}$. Together with Lemma 2 this shows that

$$
\left.\mathrm{E}^{*}|| \bar{u}_{t}^{*}\left|-\mathrm{E}^{*}\right| \bar{u}_{t}\right|^{a}=O_{p}(1)
$$

As

$$
\mathrm{E}^{*}\left|\bar{u}_{t}\right|=\mathrm{E}^{*}\left|\sum_{j=0}^{\infty} \bar{\Psi}_{j}^{*} \varepsilon_{t-j}^{*}\right| \leq\left|\sum_{j=0}^{\infty} \bar{\Psi}_{j}^{*}\right| \mathrm{E}^{*}\left|\varepsilon_{t}^{*}\right|=O_{p}(1)
$$

this proves that (28) holds. This concludes the proof of this theorem.

Next we need several lemmas in order to show the limiting distribution of the bootstrap test statistic.

Lemma 4. Let $\xi_{t}^{*}$ be the bootstrap equivalent of $\xi$ defined in equation (5), i.e.

$$
y_{t}^{*}=\pi_{0}^{* \prime} \Delta x_{t}^{*}+\sum_{j=1}^{\infty} \pi^{* \prime} \Delta z_{t-j}^{*}+\xi_{t}^{*}
$$


Then, if Assumptions 2 and $4^{\prime}$ hold,

$$
n^{-1 / 2} \sum_{t=1}^{[n r]} \xi_{t}^{*} \stackrel{d^{*}}{\longrightarrow} B_{\xi}(r)
$$

where $B_{\xi}(r)$ is a scalar Brownian motion with variance $\omega^{2}$, i.e $B_{\xi}(r)=\omega W_{1}(r)$, where $W_{1}(r)$ is the first element of the standard Brownian motion $W(r)$.

Proof of Lemma 4. Follows immediately from Theorem 1.

Lemma 5. Let $f^{*}$ denote the spectral density and $\Gamma^{*}(k)$ the autocovariance function of $u_{t}^{*}$. Under Assumptions 2 and 4', we have

$$
\sup _{\lambda}\left|f^{*}(\lambda)-f(\lambda)\right|=o_{p}^{*}(1)
$$

and

$$
\sum_{k=-\infty}^{\infty} \Gamma^{*}(k)=\sum_{k=-\infty}^{\infty} \Gamma(k)+o_{p}^{*}(1)
$$

Proof of Lemma 5. The spectral density $f^{*}(\lambda)$ of $u_{t}^{*}$ is

$$
f^{*}(\lambda)=\frac{1}{2 \pi}\left(I-\sum_{j=1}^{q} \hat{\Phi}_{j} e^{-\mathrm{i} j v}\right)^{-1} \Sigma^{*}\left(I-\sum_{j=1}^{q} \hat{\Phi}_{j} e^{-\mathrm{i} j v}\right)^{\prime-1} .
$$

Note that by Lemma $3 \Sigma^{*} \stackrel{p}{\rightarrow} \Sigma$. Furthermore,

$$
\begin{aligned}
& \left|\left(I-\sum_{j=1}^{q} \hat{\Phi}_{j} e^{-\mathrm{i} j v}\right)-\left(I-\sum_{j=1}^{q} \Phi_{j} e^{-\mathrm{i} j v}\right)\right|=\left|\sum_{j=1}^{q}\left(\hat{\Phi}_{j}-\Phi_{j}\right) e^{-\mathrm{i} j v}\right| \\
& \quad \leq \sum_{j=1}^{q}\left|\hat{\Phi}_{j}-\Phi_{q, j}\right|\left|e^{-\mathrm{i} j v}\right|+\sum_{j=1}^{q}\left|\Phi_{q, j}-\Phi_{j}\right|\left|e^{-\mathrm{i} j v}\right| \\
& \quad \leq q \max _{1 \leq j \leq q}\left|\hat{\Phi}_{j}-\Phi_{q, j}\right|+\sum_{j=1}^{q}\left|\Phi_{q, j}-\Phi_{j}\right|=o_{p}(1)
\end{aligned}
$$

by (19) and (20). Now the result in (33) follows straightforwardly.

The result in (34) follows trivially by noting that $\sum_{k=-\infty}^{\infty} \Gamma(k)=2 \pi f(0)$ and correspondingly $\sum_{k=-\infty}^{\infty} \Gamma^{*}(k)=2 \pi f^{*}(0)$.

Now we can derive the limiting distributions of the different elements of the test statistic. First define $w_{p, t}=\left(\Delta x_{t}^{\prime}, \Delta z_{t-1}^{\prime}, \ldots, \Delta z_{t-p}\right)^{\prime}$, and let $W_{p}=\left(w_{p, 1}, \ldots, w_{p, n}\right)^{\prime}, Z_{-1}=$ 
$\left(z_{0}, \ldots, z_{n-1}\right)^{\prime}, \xi_{p}=\left(\xi_{p, 1}, \ldots, \xi_{p, n}\right)^{\prime}$ and $\Delta Y=\left(\Delta y_{1}, \ldots, \Delta y_{n}\right)^{\prime}$, and define their bootstrap versions accordingly.

Lemma 6. Under Assumptions 2, 3 and $4^{\prime}$ we have
a) $n^{-2} Z_{-1}^{* \prime} Z_{-1}^{*}=n^{-2} \sum_{t=1}^{n} z_{t-1}^{*} z_{t-1}^{* \prime} \stackrel{d^{*}}{\longrightarrow} \int_{0}^{1} B(r) B(r)^{\prime} d r$
b) $n^{-1} Z_{-1}^{* \prime} \xi_{p}^{*}=n^{-1} \sum_{t=1}^{n} z_{t-1}^{*} \xi_{p, t}^{*} \stackrel{d^{*}}{\longrightarrow} \int_{0}^{1} B(r) d B_{\xi}(r)$
c) $\left\|\left(n^{-1} W_{p}^{* \prime} W_{p}^{*}\right)^{-1}\right\|=\left\|\left(\frac{1}{n} \sum_{t=1}^{n} w_{p, t}^{*} w_{p, t}^{* \prime}\right)^{-1}\right\|=O_{p}^{*}(1)$
d) $\left|Z_{-1}^{* \prime} W_{p}^{*}\right|=\left|\sum_{t=1}^{n} z_{t-1}^{*} w_{p, t}^{* \prime}\right|=O_{p}^{*}\left(n p^{1 / 2}\right)$
e) $\left|W_{p}^{* \prime} \xi_{p}^{*}\right|=\left|\sum_{t=1}^{n} w_{p, t}^{*} \xi_{p, t}^{*}\right|=O_{p}^{*}\left(n^{1 / 2} p^{1 / 2}\right)$.

Proof of Lemma 6. First we look at a). As we set $z_{0}^{*}=0$, we have

$$
z_{t}^{*}=\sum_{i=1}^{t} u_{t}^{*}
$$

and therefore

$$
B_{n}^{*}(r)=n^{-1 / 2} z_{[n r]} .
$$

Then by Theorem 2 and the continuous mapping theorem we have

$$
\begin{aligned}
n^{-2} \sum_{t=1}^{n} z_{t-1}^{*} z_{t-1}^{* \prime} & =n^{-1} \sum_{t=1}^{n} \int_{(t-1) / n}^{t / n} z_{[n r]}^{*} z_{[n r]}^{* \prime} d r=\sum_{t=1}^{n} \int_{(t-1) / n}^{t / n} B_{n}^{*}(r) B_{n}^{* \prime}(r) d r \\
& =\int_{0}^{1} B_{n}^{*}(r) B_{n}^{* \prime}(r) d r \stackrel{d^{*}}{\longrightarrow} \int_{0}^{1} B(r) B(r)^{\prime} d r
\end{aligned}
$$

as in Chang et al. (2006, Proof of Lemma 3.4).

Next we look at b). We have

$$
\left|n^{-1} \sum_{t=1}^{n} z_{t-1}^{*} \xi_{p, t}^{*}\right| \leq\left|n^{-1} \sum_{t=1}^{n} z_{t-1}^{*} \xi_{t}^{*}\right|+\left|n^{-1} \sum_{t=1}^{n} z_{t-1}^{*}\left(\xi_{p, t}^{*}-\xi_{t}^{*}\right)\right| .
$$

Hence, we first have to show that $n^{-1} \sum_{t=1}^{n} z_{t-1}^{*}\left(\xi_{p, t}^{*}-\xi_{t}^{*}\right)=o_{p}(1)$. We can follow Chang et al. (2006, Proof of Lemma A.6) for the proof. 
Define $\eta_{t}^{*}=\sum_{i=1}^{t} \varepsilon_{t}^{*}$, such that we can write

$$
z_{t}^{*}=\hat{\Psi}(1) \eta_{t}^{*}+\left(\bar{u}_{0}^{*}-\bar{u}_{t}^{*}\right)
$$

Then we have

$$
\begin{aligned}
\sum_{t=1}^{n} z_{t-1}^{*}\left(\xi_{p, t}^{*}-\xi_{t}^{*}\right)^{\prime} & =\hat{\Psi}(1) \eta_{t-1}^{*}\left(\xi_{p, t}^{*}-\xi_{t}^{*}\right)^{\prime}+\bar{u}_{0}^{*}\left(\xi_{p, t}^{*}-\xi_{t}^{*}\right)^{\prime}-\bar{u}_{t-1}^{*}\left(\xi_{p, t}^{*}-\xi_{t}^{*}\right)^{\prime} \\
& =R_{1 n}^{*}+R_{2 n}^{*}+R_{3 n}^{*}
\end{aligned}
$$

Also define $\hat{\Psi}_{p, j}$ such that

$$
\begin{aligned}
\xi_{t}^{*}-\xi_{p, t}^{*} & =\sum_{k=p+1}^{\infty} \hat{\pi}_{k}^{\prime} u_{t-k}^{*}=\sum_{k=p+1}^{\infty} \hat{\pi}_{k}^{\prime} \sum_{i=0}^{\infty} \hat{\Psi}_{i} \varepsilon_{t-k-i}^{*} \\
& =\sum_{j=p+1}^{\infty} \sum_{k=p+1}^{j} \hat{\pi}_{k}^{\prime} \hat{\Psi}_{j-k} \varepsilon_{t-j}^{*}=\sum_{j=p+1}^{\infty} \hat{\Psi}_{p, j} \varepsilon_{t-j}^{*},
\end{aligned}
$$

We then have that

$$
\begin{aligned}
\sum_{j=p+1}^{\infty}\left|\hat{\Psi}_{p, j}\right| & =\sum_{j=p+1}^{\infty}\left|\sum_{k=p+1}^{j} \hat{\pi}_{k}^{\prime} \hat{\Psi}_{j-k}\right| \leq \sum_{k=p+1}^{j}\left|\hat{\pi}_{k}^{\prime}\right| \sum_{j=p+1}^{\infty}\left|\hat{\Psi}_{j-k}\right| \\
& \leq\left(\sum_{j=p+1}^{\infty}\left|\hat{\pi}_{j}\right|\right)\left(\sum_{i=0}^{\infty}\left|\hat{\Psi}_{i}\right|\right)=\left(\sum_{j=p+1}^{\infty}\left|\hat{\pi}_{j}\right|\right) O_{p}(1) .
\end{aligned}
$$

We first want to show that $R_{1 n}^{*}=o_{p}^{*}(n)$. Let $\delta_{i j}$ be the Kronecker delta. We have

$$
\begin{aligned}
\left|\sum_{t=1}^{n} \eta_{t-1}^{*}\left(\xi_{p, t}^{*}-\xi_{t}^{*}\right)\right| & =\left|\sum_{t=1}^{n} \eta_{t-1}^{*}\left(\sum_{j=p+1}^{\infty} \hat{\Psi}_{p, j} \varepsilon_{t-j}^{*}\right)\right|=\left|\sum_{t=1}^{n} \sum_{i=1}^{t-1} \varepsilon_{i}^{*}\left(\sum_{j=p+1}^{\infty} \hat{\Psi}_{p, j} \varepsilon_{t-j}^{*}\right)\right| \\
& =\left|\sum_{t=1}^{n} \sum_{i=1}^{t-1} \sum_{j=p+1}^{\infty} \varepsilon_{i}^{*} \varepsilon_{t-j}^{* \prime} \hat{\Psi}_{p, j}^{\prime}\right|=\left|\sum_{j=p+1}^{\infty} \sum_{t=1}^{n} \sum_{i=1}^{t-1} \varepsilon_{t-i}^{*} \varepsilon_{t-j}^{* \prime} \hat{\Psi}_{p, j}^{\prime}\right| \\
& =\left|\sum_{j=p+1}^{n-1}(n-j) \Sigma \hat{\Psi}_{p, j}^{\prime}+\sum_{j=p+1}^{\infty} \sum_{t=1}^{n} \sum_{i=1}^{t-1}\left(\varepsilon_{t-i}^{*} \varepsilon_{t-j}^{* \prime}-\delta_{i j} \Sigma\right) \hat{\Psi}_{p, j}^{\prime}\right| \\
& =\sum_{j=p+1}^{\infty}\left|\hat{\Psi}_{p, j}\right| O_{p}^{*}(n)+\sum_{j=p+1}^{\infty}\left|\hat{\Psi}_{p, j}\right| O_{p}^{*}\left(n^{1 / 2}\right) \\
& =\left(\sum_{j=p+1}^{\infty}\left|\hat{\pi}_{j}\right|\right) O_{p}^{*}(n),
\end{aligned}
$$


which shows that $R_{1 n}^{*}=o_{p}^{*}(n)$.

Next we turn to $R_{2 n}^{*}$. We have

$$
\begin{aligned}
\sum_{t=1}^{n}\left(\xi_{p, t}^{*}-\xi_{t}^{*}\right) & =\sum_{t=1}^{n} \sum_{j=p+1}^{\infty} \hat{\Psi}_{p, j} \varepsilon_{t-j}^{*}=\sum_{j=p+1}^{\infty} \hat{\Psi}_{p, j} \sum_{t=1}^{n} \varepsilon_{t-j}^{*} \\
& =\left(\sum_{j=p+1}^{\infty}\left|\hat{\Psi}_{p, j}\right|\right) O_{p}^{*}\left(n^{1 / 2}\right)=\left(\sum_{j=p+1}^{\infty}\left|\hat{\pi}_{j}\right|\right) O_{p}^{*}\left(n^{1 / 2}\right),
\end{aligned}
$$

from which we can easily see that $R_{2 n}^{*}=o_{p}^{*}\left(n^{1 / 2}\right)$.

Finally we look at $R_{3 n}^{*}$. First note that by applying the Beveridge-Nelson decomposition in a slightly different way than before, we can derive that $\bar{u}_{t}=\sum_{j=0}^{\infty} \sum_{i=j+1}^{\infty} \hat{\Psi}_{j} \varepsilon_{t-j}^{*}$. Then we can write $\left|R_{3 n}^{*}\right|$ as

$$
\begin{aligned}
\left|\sum_{t=1}^{n} \bar{u}_{t-1}^{*}\left(\xi_{p, t}^{*}-\xi_{t}^{*}\right)\right| & =\left|\sum_{t=1}^{n} \bar{u}_{t-1}^{*} \sum_{j=p+1}^{\infty} \hat{\Psi}_{p, j} \varepsilon_{t-j}^{*}\right|=\left|\sum_{t=1}^{n} \sum_{i=0}^{\infty} \sum_{k=i+1}^{\infty} \hat{\Psi}_{k} \varepsilon_{t-i-1}^{*} \sum_{j=p+1}^{\infty} \hat{\Psi}_{p, j} \varepsilon_{t-j}^{*}\right| \\
& =\left|\sum_{i=0}^{\infty} \sum_{j=p+1}^{\infty} \sum_{k=i}^{\infty} \hat{\Psi}_{k} \sum_{t=1}^{n}\left(\varepsilon_{t-i-1}^{*} \varepsilon_{t-j}^{* \prime}\right) \hat{\Psi}_{p, j}^{\prime}\right| \\
& =\left|n \sum_{j=p+1}^{\infty} \sum_{k=j}^{\infty} \hat{\Psi}_{k} \Sigma \hat{\Psi}_{p, j}^{\prime}+\sum_{i=0}^{\infty} \sum_{k=i}^{\infty} \hat{\Psi}_{k} \sum_{j=p+1}^{\infty} \sum_{t=1}^{n}\left(\varepsilon_{t-i-1}^{*} \varepsilon_{t-j}^{*}-\delta_{i+1, j} \Sigma\right) \hat{\Psi}_{p, j}^{\prime}\right| \\
& =\sum_{j=p+1}^{\infty} \sum_{k=j}^{\infty}\left|\hat{\Psi}_{k}\right|\left|\hat{\Psi}_{p, j}\right| O_{p}^{*}(n)+\sum_{i=0}^{\infty} \sum_{k=i}^{\infty}\left|\hat{\Psi}_{k}\right| \sum_{j=p+1}^{\infty}\left|\hat{\Psi}_{p, j}\right| O_{p}^{*}\left(n^{1 / 2}\right) \\
& =\sum_{j=p+1}^{\infty}\left|\hat{\Psi}_{p, j}\right| O_{p}^{*}(n)+\sum_{j=p+1}^{\infty}\left|\hat{\Psi}_{p, j}\right| O_{p}^{*}\left(n^{1 / 2}\right) \\
& =\left(\sum_{j=p+1}^{\infty}\left|\hat{\pi}_{j}\right|\right) O_{p}^{*}(n) .
\end{aligned}
$$

Therefore, $R_{3 n}^{*}=o_{p}^{*}(n)$, and hence

$$
n^{-1} \sum_{t=1}^{n} z_{t-1}^{*}\left(\xi_{p, t}^{*}-\xi_{t}^{*}\right)=n^{-1}\left(R_{1 n}^{*}+R_{2 n}^{*}+R_{3 n}^{*}\right)=o_{p}^{*}(1) .
$$

Then

$$
n^{-1} \sum_{t=1}^{n} z_{t-1}^{*} \xi_{p, t}^{*}=n^{-1} \sum_{t=1}^{n} z_{t-1}^{*} \xi_{t}^{*}+o_{p}^{*}(1),
$$


while by Park and Phillips (1989, Lemma 2.1), Theorem 2 and Lemma 4, we have that

$$
n^{1} \sum_{t=1}^{n} z_{t-1}^{*} \xi_{t}^{*} \stackrel{d^{*}}{\longrightarrow} \int_{0}^{1} B(r) d B_{\xi}(r)
$$

This completes the proof of part b).

For c), we want to show that

$$
\mathrm{E}^{*}\left\|\left(\frac{1}{n} \sum_{t=1}^{n} w_{p, t}^{*} w_{p, t}^{* \prime}\right)^{-1}\right\|=O_{p}(1) .
$$

Following Chang and Park (2003, Proof of Lemma 3) we first want to show that

$$
\mathrm{E}^{*}\left|\sum_{t=1}^{n}\left[u_{t-i}^{*} u_{t-j}^{* \prime}-\Gamma^{*}(i-j)\right]\right|^{2}=O_{p}(n) .
$$

For this to hold it is sufficient to show that

$$
\mathrm{E}^{*}\left(\sum_{t=1}^{n}\left[u_{a, t-i}^{*} u_{b, t-j}^{* \prime}-\Gamma_{a b}^{*}(i-j)\right]\right)^{2}=O_{p}(n),
$$

for all $1 \leq a, b \leq 1+l$, where $u_{a, t}$ is the $a$-th element of $u_{t}$, and similarly $\Gamma_{a b}(i-j)$ is the $(a, b)$-th element of $\Gamma(i-j)$.

Analogous to the case for univariate time series models discussed in Berk (1974, eqs (2.10) and (2.11), p. 491), we have that

$$
\begin{aligned}
& \mathrm{E}\left(\sum_{t=1}^{n}\left[u_{a, t-i}^{*} u_{b, t-i}^{*}-\Gamma_{a b}^{*}(i-j)\right]\right)^{2} \leq 2 n \sum_{k=-\infty}^{\infty} \Gamma_{a b}^{*}(k)^{2} \\
& \quad+n \sum_{c, d, e, f}\left|\tilde{\kappa}_{c d e f}^{*}\right|\left(\sum_{k=0}^{\infty} \Psi_{a c, k}^{* 2}\right)^{1 / 2}\left(\sum_{k=0}^{\infty} \Psi_{b d, k}^{* 2}\right)^{1 / 2}\left(\sum_{k=0}^{\infty} \Psi_{a e, k}^{* 2}\right)^{1 / 2}\left(\sum_{k=0}^{\infty} \Psi_{b f, k}^{* 2}\right)^{1 / 2},
\end{aligned}
$$

where $\tilde{\kappa}_{c d e f}^{*}=\mathrm{E}\left(\varepsilon_{c, t}^{*} \varepsilon_{d, t}^{*} \varepsilon_{e, t}^{*} \varepsilon_{f, t}^{*}\right)-\sigma_{c d} \sigma_{e f}-\sigma_{c e} \sigma_{d f}-\sigma_{c f} \sigma_{d e}$ and $\sigma_{c d}=\mathrm{E}\left(\varepsilon_{c, t}^{*} \varepsilon_{d, t}^{*}\right)$. Note that $\left|\tilde{\kappa}_{c d e f}^{*}\right|=O_{p}(1)$ as $\mathrm{E}\left|\varepsilon_{t}^{*}\right|^{4}=O_{p}(1)$ (take $a=4$ in Lemma 2). Furthermore, $\sum_{k=-\infty}^{\infty} \Gamma_{a b}^{*}(k)^{2}=$ $O_{p}(1)$ through Lemma 5 and $\left(\sum_{k=0}^{\infty} \Psi_{a c, k}^{* 2}\right)^{1 / 2}=O_{p}(1)$ as $\sum_{k=0}^{\infty} k^{1 / 2}\left|\hat{\Psi}_{k}\right|=O_{p}(1)$, which we demonstrated in the proof of Theorem 2, equation (30). Now equation (43) follows straightforwardly.

Next, partition $\Gamma^{*}(k)$ as

$$
\Gamma^{*}(k)=\left[\begin{array}{ll}
\Gamma_{11}^{*}(k) & \Gamma_{12}^{*}(k) \\
\Gamma_{21}^{*}(k) & \Gamma_{22}^{*}(k)
\end{array}\right]
$$


and define $\Gamma_{2 \cdot}(k)=\left[\Gamma_{21}^{*}(k), \Gamma_{22}^{*}(k)\right]$ and $\Gamma_{\cdot 2}(k)=\left[\Gamma_{12}^{*}(k)^{\prime}, \Gamma_{22}^{*}(k)^{\prime}\right]^{\prime}$. Then define $\Omega_{p p}^{*}$ as

$$
\Omega_{p p}^{*}=\left[\begin{array}{cccc}
\Gamma_{22}^{*}(0) & \Gamma_{2 \cdot}^{*}(-1) & \ldots & \Gamma_{2 \cdot}^{*}(-p) \\
\Gamma_{\cdot 2}^{*}(1) & \Gamma^{*}(0) & \ldots & \Gamma^{*}(1-p) \\
\vdots & \vdots & \ddots & \vdots \\
\Gamma_{\cdot 2}^{*}(p) & \Gamma^{*}(p-1) & \ldots & \Gamma^{*}(0)
\end{array}\right]
$$

Furthermore, for a vector $v$, let $v(i, j)$ (with $i<j$ ) indicate the subvector from elements $i$ to $j$, i.e. $v(i, j)=\left(v_{i}, \ldots, v_{j}\right)^{\prime}$. Similarly, for a square matrix $M$ let $M(i, j)$ be the submatrix consisting of the block of elements between rows $i$ and $j$ and columns $i$ and $j$, i.e. $M(i, j)=$ $\left(M_{i}(i, j), \ldots, M_{j}(i, j)\right)$, where $M_{i}$ is the $i$-th column vector in $M$. Finally, define $k_{j}$ as

$$
k_{j}=\left\{\begin{array}{cc}
0 & \text { if } j=0, \\
l & \text { if } j=1, \\
(l+1) j & \text { if } j=2, \ldots, p+1 .
\end{array}\right.
$$

As $\|M\|^{2} \leq \sum_{i, j}\left\|M_{i j}\right\|^{2},{ }^{7}$ we can write

$$
\begin{aligned}
\mathrm{E}^{*}\left\|\frac{1}{n} \sum_{t=1}^{n} w_{p, t}^{*} w_{p, t}^{* \prime}-\Omega_{p p}^{*}\right\|^{2} \leq & \mathrm{E}^{*}\left(\sum_{(i=0, j=0)}^{(p, p)}\left\|w_{p, t}^{*}\left(k_{i}+1, k_{j}\right) w_{p, t}^{*}\left(k_{i}+1, k_{j}\right)^{\prime}-\Omega_{p p}^{*}\left(k_{i}+1, k_{j}\right)\right\|^{2}\right) \\
\leq & \mathrm{E}^{*}\left(\left\|\frac{1}{n} \sum_{t=1}^{n} u_{2, t}^{*} u_{2, t}^{* \prime}-\Gamma_{22}(0)\right\|\left\|^{2}+\sum_{j=1}^{p}\right\| \frac{1}{n} \sum_{t=1}^{n} u_{2, t}^{*} u_{t-j}^{* \prime}-\Gamma_{2 \cdot}(-j)\|\|^{2}\right. \\
& \left.+\sum_{i=1}^{p}\left\|\frac{1}{n} \sum_{t=1}^{n} u_{t-i}^{*} u_{2, t}^{* \prime}-\Gamma_{.2}(i)\right\|^{2}+\sum_{(i=1, j=1)}^{(p, p)}\left\|\frac{1}{n} \sum_{t=1}^{n} u_{t-i}^{*} u_{t-j}^{* \prime}-\Gamma(j-i)\right\|^{2}\right) \\
= & O_{p}\left(n^{-1}\right)+O_{p}\left(n^{-1} p\right)+O_{p}\left(n^{-1} p\right)+O_{p}\left(n^{-1} p^{2}\right)=O_{p}\left(n^{-1} p^{2}\right) .
\end{aligned}
$$

Next we need to show that

$$
\left\|\Omega_{p p}^{*-1}\right\| \leq\left[2 \pi\left(\inf _{\lambda} f^{*}(\lambda)\right)\right]^{-1}=O_{p}(1) .
$$

Let us consider an "extended" $\Omega_{p p}^{*}$ matrix, i.e.

$$
\tilde{\Omega}_{p p}^{*}=\left[\begin{array}{ccccc}
\Gamma_{11}^{*}(0) & \Gamma_{12}^{*}(0) & \Gamma_{1 .}^{*}(-1) & \ldots & \Gamma_{1 .}^{*}(-p) \\
\Gamma_{21}^{*}(0) & & & & \\
\Gamma_{\cdot .}^{*}(1) & & \Omega_{p p}^{*} & & \\
\vdots & & & & \\
\Gamma_{.1}^{*}(p) & & & &
\end{array}\right]=\left[\begin{array}{cccc}
\Gamma^{*}(0) & \Gamma^{*}(-1) & \ldots & \Gamma^{*}(-p) \\
\Gamma^{*}(1) & \Gamma^{*}(0) & \ldots & \Gamma^{*}(1-p) \\
\vdots & \vdots & \ddots & \vdots \\
\Gamma^{*}(p) & \Gamma^{*}(p-1) & \ldots & \Gamma^{*}(0)
\end{array}\right]
$$

\footnotetext{
${ }^{7}$ We let $M_{i j}$ denote submatrices into which one can partition $M$.
} 
Let $\lambda^{*}=\left(\lambda_{1}^{*}, \ldots, \lambda_{(l+1) p}^{*}\right)^{\prime}$ be the eigenvalues of $\tilde{\Omega}_{p p}^{*}$ and define $0<F_{1}^{*}=\inf _{\lambda}\left\|f^{*}(\lambda)\right\|$. Then as a direct consequence of Lemma A.2 of Chang et al. (2006) we have that

$$
\left\|\Omega_{p p}^{*-1}\right\| \leq\left(2 \pi F_{1}^{*}\right)^{-1}=O_{p}(1)
$$

As $\left\|\Omega_{p p}^{*-1}\right\| \leq\left\|\tilde{\Omega}_{p p}^{*-1}\right\|,{ }^{8}$ we know that $\left\|\Omega_{p p}^{*-1}\right\|=O_{p}(1)$ as well. Then

$$
\begin{aligned}
\left\|\left(\frac{1}{n} \sum_{t=1}^{n} w_{p, t}^{*} w_{p, t}^{* \prime}\right)^{-1}\right\| & \leq\left\|\Omega_{p p}^{*-1}\right\|+\left\|\left(\frac{1}{n} \sum_{t=1}^{n} w_{p, t}^{*} w_{p, t}^{* \prime}\right)^{-1}-\Omega_{p p}^{*-1}\right\| \\
& =\left\|\Omega_{p p}^{*-1}\right\|+\left\|\Omega_{p p}^{*-1}\left(\Omega_{p p}^{*}-\frac{1}{n} \sum_{t=1}^{n} w_{p, t}^{*} w_{p, t}^{* \prime}\right)\left(\frac{1}{n} \sum_{t=1}^{n} w_{p, t}^{*} w_{p, t}^{* \prime}\right)^{-1}\right\| \\
& \leq\left\|\Omega_{p p}^{*-1}\right\|+\left\|\Omega_{p p}^{*-1}\right\|\left\|\frac{1}{n} \sum_{t=1}^{n} w_{p, t}^{*} w_{p, t}^{* \prime}-\Omega_{p p}^{*}\right\|\left\|\left(\frac{1}{n} \sum_{t=1}^{n} w_{p, t}^{*} w_{p, t}^{* \prime}\right)^{-1}\right\| \\
& \leq \frac{\left\|\Omega_{p p}^{*-1}\right\|}{1-\left\|\Omega_{p p}^{*-1}\right\|\left\|\frac{1}{n} \sum_{t=1}^{n} w_{p, t}^{*} w_{p, t}^{* \prime}-\Omega_{p p}^{*}\right\|},
\end{aligned}
$$

which holds for large $n$ as $\left\|\frac{1}{n} \sum_{t=1}^{n} w_{p, t}^{*} w_{p, t}^{* \prime}-\Omega_{p p}^{*}\right\|=O_{p}\left(n^{-1} p^{2}\right)$. As $\left\|\Omega_{p p}^{*-1}\right\|=O_{p}(1)$, the result in (41) follows.

For d) we want to show that

$$
\mathrm{E}^{*}\left|\sum_{t=1}^{n} z_{t-1}^{*} w_{p, t}^{* \prime}\right|=O_{p}\left(n p^{1 / 2}\right) .
$$

Following Chang and Park (2002, Proof of Lemma 3.2), we write

$$
\sum_{t=1}^{n} z_{t-1}^{*} u_{t-j}^{* \prime}=\sum_{t=1}^{n} z_{t-1}^{*} u_{t}^{* \prime}+R_{n}^{*}
$$

\footnotetext{
${ }^{8}$ Suppose we have a matrix $M$ and a vector $v$ that we can write as

$$
M=\left[\begin{array}{ll}
M_{11} & M_{12} \\
M_{21} & M_{22}
\end{array}\right] \quad v=\left[\begin{array}{l}
v_{1} \\
v_{2}
\end{array}\right] .
$$
}

Then we have that

$$
\begin{aligned}
& \|M\|^{2}=\max _{v}|M v|^{2} /|v|^{2}=\max _{v_{1}, v_{2}}\left|\begin{array}{l}
M_{11} v_{1}+M_{12} v_{2} \\
M_{21} v_{1}+M_{22} v_{2}
\end{array}\right|^{2} /\left|\begin{array}{l}
v_{1} \\
v_{2}
\end{array}\right|^{2} \\
& =\max _{v_{1}, v_{2}} \frac{\left|M_{11} v_{1}+M_{12} v_{2}\right|^{2}+\left|M_{21} v_{1}+M_{22} v_{2}\right|^{2}}{\left|v_{1}\right|^{2}+\left|v_{2}\right|^{2}} \geq \max _{v_{1}, v_{2}} \frac{\left|M_{11} v_{1}\right|^{2}+\left|M_{12} v_{2}\right|^{2}+\left|M_{21} v_{1}\right|^{2}+\left|M_{22} v_{2}\right|^{2}}{\left|v_{1}\right|^{2}+\left|v_{2}\right|^{2}} \\
& \geq \max _{v_{2}\left(v_{1}=0\right)} \frac{\left|M_{12} v_{2}\right|^{2}+\left|+M_{22} v_{2}\right|^{2}}{\left|v_{2}\right|^{2}} \geq \max _{v_{2}} \frac{\left|M_{22}\right|^{2}}{\left|v_{2}\right|^{2}}=\left\|M_{22}\right\|^{2} .
\end{aligned}
$$


where

$$
R_{n}^{*}=\sum_{t=1}^{n} z_{t-1}^{*} u_{t-j}^{* \prime}-\sum_{t=1}^{n} z_{t-1}^{*} u_{t}^{* \prime}
$$

and we want to show that $R_{n}^{*}=O_{p}^{*}(n)$ uniformly in $j$.

First, write

$$
\sum_{t=1}^{n} z_{t-1}^{*} u_{t}^{* \prime}=\sum_{t=1}^{n-j} z_{t-1}^{*} u_{t}^{* \prime}+\sum_{t=n-j+1}^{n} z_{t-1}^{*} u_{t}^{* \prime}=\sum_{t=1}^{n} z_{t-j-1}^{*} u_{t-j}^{* \prime}+\sum_{t=n-j+1}^{n} z_{t-1}^{*} u_{t}^{* \prime}
$$

(as $u_{t}^{*}=0$ for $t=0, \ldots,-p$ ) and rewrite $R_{n}^{*}$ as

$$
\sum_{t=1}^{n}\left(z_{t-1}^{*}-z_{t-j-1}^{*}\right) u_{t-j}^{* \prime}-\sum_{t=n-j+1}^{n} z_{t-1}^{*} u_{t}^{\prime}=R_{1 n}^{*}-R_{2 n}^{*} .
$$

Then

$$
\begin{aligned}
R_{1 n}^{*} & =\sum_{t=1}^{n}\left(z_{t-1}^{*}-z_{t-j-1}^{*}\right) u_{t-j}^{* \prime}=\sum_{t=1}^{n}\left(\sum_{i=1}^{j} u_{t-i}^{*}\right) u_{t-j}^{* \prime}=\sum_{i=1}^{j} \sum_{t=1}^{n} u_{t-i}^{*} u_{t-j}^{* \prime} \\
& =n \sum_{i=1}^{j} \Gamma^{*}(i-j)+\sum_{i=1}^{j}\left[\sum_{t=1}^{n}\left(u_{t-i}^{*} u_{t-j}^{* \prime}-\Gamma^{*}(i-j)\right)\right] \\
& =O_{p}(n)+O_{p}^{*}\left(n^{1 / 2} p\right)
\end{aligned}
$$

as $1 \leq j \leq p$, the result in equation (42), and the fact that $\sum_{k=-\infty}^{\infty} \Gamma^{*}(k)=O_{p}(1)$ by Assumption 2 and Lemma 5. We can write $R_{2 n}^{*}$ as

$$
\begin{aligned}
R_{2 n}^{*} & =\sum_{t=n-j+1}^{n}\left(\sum_{i=1}^{t-1} u_{t-i}^{*}\right) u_{t}^{* \prime}=\sum_{t=n-j+1}^{n} \sum_{i=1}^{t-1} u_{t-i}^{*} u_{t}^{* \prime}=\sum_{t=n-j+1}^{n}\left(\sum_{i=1}^{n-j} u_{t-i}^{*}+\sum_{i=n-j+1}^{t-1} u_{t-i}^{*}\right) u_{t}^{* \prime} \\
& =\sum_{t=n-j+1}^{n} \sum_{i=1}^{n-j} u_{t-i}^{*} u_{t}^{* \prime}+\sum_{t=n-j+2}^{n} \sum_{i=n-j+1}^{t-1} u_{t-i}^{*} u_{t}^{* \prime}=R_{2 n}^{a *}+R_{2 n}^{b *} .
\end{aligned}
$$

Then we have

$$
R_{2 n}^{a *}=j \sum_{i=1}^{n-j} \Gamma^{*}(i)+\sum_{t=n-j+1}^{n}\left[\sum_{i=1}^{n-j}\left(u_{t-i}^{*} u_{t}^{* \prime}-\Gamma(i)^{*}\right)\right]=O_{p}(p)+O_{p}^{*}\left(n^{1 / 2} p\right)
$$

and

$$
R_{2 n}^{b *}=(j-1) \sum_{i=n-j+1}^{t-j} \Gamma^{*}(i)+\sum_{t=n-j+2}^{n}\left[\sum_{i=n-j+1}^{t-1}\left(u_{t-i}^{*} u_{t}^{* \prime}-\Gamma(i)^{*}\right)\right]=O_{p}(p)+O_{p}^{*}\left(p^{3 / 2}\right)
$$


as

$$
\begin{aligned}
\sum_{t=n-j+2}^{n}\left[\sum_{i=n-j+1}^{t-1}\left(u_{t-i}^{*} u_{t}^{* \prime}-\Gamma(i)^{*}\right)\right] & =\sum_{t=n-j+2}^{n}\left[\sum_{i=1}^{t-(n-j)-1}\left(u_{t-n+j-i}^{*} u_{t}^{* \prime}-\Gamma(i)^{*}\right)\right] \\
& =\sum_{t=n-j+2}^{n} O_{p}^{*}\left((t-n+j)^{1 / 2}\right)=\sum_{t=n-j+2}^{n} O_{p}^{*}\left(j^{1 / 2}\right) \\
& =\sum_{t=n-j+2}^{n} O_{p}^{*}\left(p^{1 / 2}\right)=(j-1) O_{p}^{*}\left(p^{1 / 2}\right)=O_{p}^{*}\left(p^{3 / 2}\right) .
\end{aligned}
$$

Hence,

$$
\sum_{t=1}^{n} z_{t-1}^{*} u_{t-j}^{* \prime}=\sum_{t=1}^{n} z_{t-1}^{*} u_{t}^{* \prime}+R_{n}^{*}=\sum_{t=1}^{n} z_{t-1}^{*} u_{t}^{* \prime}+O_{p}(n)+O_{p}^{*}\left(n^{1 / 2} p\right) .
$$

Note that

$$
\left|\sum_{t=1}^{n} z_{t-1}^{*} u_{t}^{* \prime}\right|=O_{p}^{*}(n)
$$

by Phillips (1988), and

$$
\left|\sum_{t=1}^{n} z_{t-1}^{*} u_{2, t}^{* \prime}\right|=\left|\sum_{t=1}^{n} z_{t-1}^{*} u_{t}^{* \prime}\right|+O_{p}^{*}(n) .
$$

Then

$$
\begin{aligned}
& \mathrm{E}^{*}\left|\sum_{t=1}^{n} z_{t-1}^{*} w_{p, t}^{* \prime}\right|=\mathrm{E}^{*}\left|\sum_{t=1}^{n}\left[\begin{array}{llll}
z_{t-1}^{*} u_{2, t}^{* \prime} & z_{t-1}^{*} u_{t-1}^{* \prime} & \ldots & z_{t-1}^{*} u_{t-p}^{* \prime}
\end{array}\right]\right| \\
& =\mathrm{E}^{*}\left(\left|\sum_{t=1}^{n} z_{t-1}^{*} u_{2, t}^{* \prime}\right|^{2}+\sum_{j=1}^{p}\left|\sum_{t=1}^{n} z_{t-1}^{*} u_{t-j}^{* \prime}\right|^{2}\right)^{1 / 2} \\
& =\mathrm{E}^{*}\left(\left|\sum_{t=1}^{n} z_{t-1}^{*} u_{2, t}^{* \prime}\right|^{2}+\sum_{j=1}^{p}\left(\left|\sum_{t=1}^{n} z_{t-1}^{*} u_{t}^{* \prime}\right|+O_{p}(n)+O_{p}^{*}\left(n^{1 / 2} p\right)\right)^{2}\right)^{1 / 2} \\
& =\mathrm{E}^{*}\left(\left(\left|\sum_{t=1}^{n} z_{t-1}^{*} u_{t}^{* \prime}\right|+O_{p}^{*}(n)\right)^{2}+p\left(\left|\sum_{t=1}^{n} z_{t-1}^{*} u_{t}^{* \prime}\right|+O_{p}(n)+O_{p}^{*}\left(n^{1 / 2} p\right)\right)^{2}\right)^{1 / 2} \\
& =O_{p}\left(n p^{1 / 2}\right) \text {. }
\end{aligned}
$$


Finally, we look at e). We want to show that

$$
\left|\sum_{t=1}^{n} w_{p, t}^{*} \xi_{p, t}^{* \prime}\right|=O_{p}^{*}\left(n^{1 / 2} p^{1 / 2}\right) .
$$

We can follow Chang et al. (2006, Proof of Lemma A.6) for the proof. Write

$$
\sum_{t=1}^{n} w_{p, t}^{*} \xi_{p, t}^{*}=\sum_{t=1}^{n} w_{p, t}^{*} \xi_{t}^{*}+\sum_{t=1}^{n} w_{t}^{*}\left(\xi_{p, t}^{*}-\xi_{t}^{*}\right) .
$$

We first show that

$$
\sum_{t=1}^{n} u_{t-j}^{*}\left(\xi_{p, t}^{*}-\xi_{t}^{*}\right)=o_{p}^{*}\left(n^{1 / 2}\right)
$$

uniformly in $1 \leq j \leq p$. We have that

$$
\begin{aligned}
\left|\sum_{t=1}^{n} u_{t-j}^{*}\left(\xi_{p, t}^{*}-\xi_{t}^{*}\right)\right| & =\left|\sum_{t=1}^{n} u_{t-j}^{*}\left(\sum_{k=p+1}^{\infty} \hat{\Psi}_{p, k} \varepsilon_{t-k}^{*}\right)\right|=\left|\sum_{t=1}^{n}\left(\sum_{i=1}^{t-j} \varepsilon_{i}\right)\left(\sum_{k=p+1}^{\infty} \hat{\Psi}_{p, k} \varepsilon_{t-k}^{*}\right)\right| \\
& =\left|\sum_{t=1}^{n} \sum_{i=1}^{t-j} \sum_{k=p+1}^{\infty} \varepsilon_{i} \varepsilon_{t-k}^{\prime} \hat{\Psi}_{p, k}^{\prime}\right|=\left|\sum_{k=p+1}^{\infty} \sum_{t=1}^{n} \sum_{i=j}^{t-1} \varepsilon_{t-i} \varepsilon_{t-k}^{\prime} \hat{\Psi}_{p, k}^{\prime}\right| \\
& =\left|\sum_{k=p+1}^{n-1}(n-k) \Sigma \hat{\Psi}_{p, k}^{\prime}+\sum_{k=p+1}^{\infty} \sum_{t=1}^{n} \sum_{i=j}^{t-1}\left(\varepsilon_{t-i}^{*} \varepsilon_{t-k}^{* \prime}-\delta_{i k} \Sigma\right) \hat{\Psi}_{p, k}^{\prime}\right| \\
& =\sum_{k=p+1}^{\infty}\left|\hat{\Psi}_{p, k}\right| O_{p}^{*}(n)+\sum_{k=p+1}^{\infty}\left|\hat{\Psi}_{p, k}\right| O_{p}^{*}\left(n^{1 / 2}\right) \\
& =\left(\sum_{k=p+1}^{\infty}\left|\hat{\pi}_{k}\right|\right) O_{p}^{*}(n)=o_{p}^{*}\left(n^{1 / 2}\right),
\end{aligned}
$$

such that

$$
\sum_{t=1}^{n} u_{t-j}^{*} \xi_{p, t}^{*}=\sum_{t=1}^{n} u_{t-j}^{*} \xi_{t}^{*}+o_{p}^{*}\left(n^{1 / 2}\right)
$$


Furthermore,

$$
\begin{aligned}
\mathrm{E}^{*}\left|\sum_{t=1}^{n} u_{t-j}^{*} \xi_{t}^{*}\right|^{2} & =\mathrm{E}^{*}\left(\sum_{s=1}^{n} u_{s-j}^{*} \xi_{s}^{*}\right)^{\prime}\left(\sum_{t=1}^{n} u_{t-j}^{*} \xi_{t}^{*}\right) \\
& =\sum_{s=1}^{n} \sum_{t=1}^{n} \mathrm{E}^{*} u_{s-j}^{* \prime} u_{t-j}^{*} \xi_{s}^{*} \xi_{t}^{*}=\sum_{s=1}^{n} \sum_{t=1}^{n} \mathrm{E}^{*} u_{s-j}^{* \prime} u_{t-j}^{*} \mathrm{E}^{*} \xi_{s}^{*} \xi_{t}^{*} \\
& =\sum_{t=1}^{n} \mathrm{E}^{*} u_{t-j}^{* \prime} u_{t-j}^{*} \mathrm{E}^{*} \xi_{t}^{* 2}=O_{p}(n) .
\end{aligned}
$$

Then

$$
\left|\sum_{t=1}^{n} w_{p, t}^{*} \xi_{p, t}^{*}\right|=\left(\sum_{j=1}^{p}\left|\sum_{t=1}^{n} u_{t-j}^{*} \xi_{p, t}^{*}\right|^{2}\right)^{1 / 2}=O_{p}^{*}\left(n^{1 / 2} p^{1 / 2}\right),
$$

which concludes the proof.

The following lemma shows the consistency of the bootstrap variance estimator.

Lemma 7. Let $\hat{\omega}^{* 2}$ be the estimator of the variance of the bootstrap errors $\xi_{p, t}^{*}$ in regression (11), i.e.

$$
\hat{\omega}^{* 2}=\frac{1}{n}\left(\Delta y^{*}-Z_{-1}^{*} \hat{\delta}^{*}\right)^{\prime}\left(I-W_{p}^{*}\left(W_{p}^{* \prime} W_{p}^{*}\right)^{-1} W_{p}^{* \prime}\right)\left(\Delta y^{*}-Z_{-1}^{*} \hat{\delta}^{*}\right)
$$

Then $\hat{\omega}^{* 2} \stackrel{p^{*}}{\longrightarrow} \omega^{2}$ under Assumptions 2, 3 and $4^{\prime}$.

Proof of Lemma \%. Note that

$$
\begin{aligned}
n \hat{\omega}^{* 2}= & \left(\Delta y^{*}-Z_{-1}^{*} \hat{\delta}^{*}\right)^{\prime}\left(I-W_{p}^{*}\left(W_{p}^{* \prime} W_{p}^{*}\right)^{-1} W_{p}^{* \prime}\right)\left(\Delta y^{*}-Z_{-1}^{*} \hat{\delta}^{*}\right) \\
= & \Delta y^{* \prime}\left(I-W_{p}^{*}\left(W_{p}^{* \prime} W_{p}^{*}\right)^{-1} W_{p}^{* \prime}\right) \Delta y^{*}-\Delta y^{* \prime}\left(I-W_{p}^{*}\left(W_{p}^{* \prime} W_{p}^{*}\right)^{-1} W_{p}^{* \prime}\right) Z_{-1}^{*} \hat{\delta}^{*} \\
& -\hat{\delta}^{* \prime} Z_{-1}^{* \prime}\left(I-W_{p}^{*}\left(W_{p}^{* \prime} W_{p}^{*}\right)^{-1} W_{p}^{* \prime}\right) \Delta y^{*}+\hat{\delta}^{* \prime} Z_{-1}^{* \prime}\left(I-W_{p}^{*}\left(W_{p}^{* \prime} W_{p}^{*}\right)^{-1} W_{p}^{* \prime}\right) Z_{-1}^{*} \hat{\delta}^{*} \\
= & \xi_{p}^{* \prime}\left(I-W_{p}^{*}\left(W_{p}^{* \prime} W_{p}^{*}\right)^{-1} W_{p}^{* \prime}\right) \xi_{p}^{*}-\xi_{p}^{* \prime}\left(I-W_{p}^{*}\left(W_{p}^{* \prime} W_{p}^{*}\right)^{-1} W_{p}^{* \prime}\right) Z_{-1}^{*} \hat{\delta}^{*} \\
& -\hat{\delta}^{* \prime} Z_{-1}^{* \prime}\left(I-W_{p}^{*}\left(W_{p}^{* \prime} W_{p}^{*}\right)^{-1} W_{p}^{* \prime}\right) \xi_{p}^{*}+\hat{\delta}^{* \prime} Z_{-1}^{* \prime}\left(I-W_{p}^{*}\left(W_{p}^{* \prime} W_{p}^{*}\right)^{-1} W_{p}^{* \prime}\right) Z_{-1}^{*} \hat{\delta}^{*} .
\end{aligned}
$$

which we write as

$$
\hat{\omega}^{* 2}=C_{n}^{*}-2 D_{n}^{*}+E_{n}^{*}
$$

We first look at $C_{n}^{*}$. Write

$$
C_{n}^{*}=n^{-1} \xi_{p}^{* \prime} \xi_{p}-n^{-1} \xi_{p}^{* \prime} W_{p}^{*}\left(W_{p}^{* \prime} W_{p}^{*}\right)^{-1} W_{p}^{* \prime} \xi_{p}^{*} .
$$


Using that $\hat{\delta}^{*}=O_{p}\left(n^{-1}\right)$ and the results from Lemma 6 , we have that

$$
\begin{aligned}
n^{-1}\left|\xi_{p}^{* \prime} W_{p}^{*}\left(W_{p}^{* \prime} W_{p}^{*}\right)^{-1} W_{p}^{* \prime} \xi_{p}^{*}\right| & \leq n^{-1}\left|n^{-1} \xi_{p}^{* \prime} W_{p}^{*}\right|||\left(W_{p}^{* \prime} W_{p}^{*}\right)^{-1}||\left|W_{p}^{* \prime} \xi_{p}^{*}\right| \\
& =n^{-1} O_{p}^{*}\left(n^{1 / 2} p^{1 / 2}\right) O_{p}^{*}\left(n^{-1}\right) O_{p}^{*}\left(n^{1 / 2} p^{1 / 2}\right)=o_{p}^{*}\left(n^{-1 / 2}\right) .
\end{aligned}
$$

Hence,

$$
C_{n}^{*}=n^{-1} \xi_{p}^{* \prime} \xi_{p}+o_{p}^{*}(1)
$$

Next we turn to $D_{n}^{*}$. We can write $D_{n}^{*}$ as

$$
D_{n}^{*}=n^{-1} \xi_{p}^{* \prime} \hat{\delta}^{*}-n^{-1} \xi_{p}^{* \prime} W_{p}^{*}\left(W_{p}^{* \prime} W_{p}^{*}\right)^{-1} W_{p}^{* \prime} Z_{-1}^{*} \hat{\delta}^{*}
$$

Again using Lemma 6 and $\hat{\delta}^{*}=O_{p}^{*}\left(n^{-1}\right)$, we have

$$
\begin{aligned}
\left|D_{n}^{*}\right| & \leq\left|\xi_{p}^{* \prime} Z_{-1}^{*}\right|\left|\hat{\delta}^{*}\right|+\left|\xi_{p}^{* \prime} W_{p}^{*}\right| \|\left(W_{p}^{* \prime} W_{p}^{*}\right)^{-1}||\left|W_{p}^{* \prime} Z_{-1}^{*}\right|\left|\hat{\delta}^{*}\right| \\
& =n^{-1} O_{p}^{*}(n) O_{p}^{*}\left(n^{-1}\right)+n^{-1} O_{p}^{*}\left(n^{1 / 2} p^{1 / 2}\right) O_{p}^{*}\left(n^{-1}\right) O_{p}^{*}\left(n p^{1 / 2}\right) O_{p}^{*}\left(n^{-1}\right)=O_{p}^{*}\left(n^{-1}\right) .
\end{aligned}
$$

Finally we look at $E_{n}^{*}$ :

$$
E_{n}^{*}=\hat{\delta}^{* \prime} Z_{-1}^{* \prime} Z_{-1}^{*} \hat{\delta}^{*}-\hat{\delta}^{* \prime} Z_{-1}^{* \prime} W_{p}^{*}\left(W_{p}^{* \prime} W_{p}^{*}\right)^{-1} W_{p}^{* \prime} Z_{-1}^{*} \hat{\delta}^{*}
$$

As before, we use the results from Lemma 6 and $\hat{\delta}^{*}=O_{p}^{*}\left(n^{-1}\right)$ to obtain

$$
\begin{aligned}
\left|E_{n}^{*}\right| & \leq n^{-1}\left|\hat{\delta}^{* \prime}\right||| Z_{-1}^{* \prime} Z_{-1}^{*}||\left|\hat{\delta}^{*}\right|+n^{-1}\left|\hat{\delta}^{* \prime}\right|\left|Z_{-1}^{* \prime} W_{p}^{*}\right|||\left(W_{p}^{* \prime} W_{p}^{*}\right)^{-1}||\left|W_{p}^{* \prime} Z_{-1}^{*}\right|\left|\hat{\delta}^{*}\right| \\
& =n^{-1} O_{p}^{*}\left(n^{-1}\right) O_{p}^{*}\left(n^{2}\right) O_{p}^{*}\left(n^{-1}\right)+n^{-1} O_{p}^{*}\left(n^{-1}\right) O_{p}^{*}\left(n p^{1 / 2}\right) O_{p}^{*}\left(n^{-1}\right) O_{p}^{*}\left(n p^{1 / 2}\right) O_{p}^{*}\left(n^{-1}\right) \\
& =O_{p}^{*}\left(n^{-1}\right) .
\end{aligned}
$$

Therefore, we have that

$$
\hat{\omega}^{* 2}=\frac{1}{n} \sum_{t=1}^{n} \xi_{p, t}^{* 2}+o_{p}^{*}(1) .
$$

Next we wish to show that $\frac{1}{n} \sum_{t=1}^{n} \xi_{p, t}^{* 2}=\frac{1}{n} \sum_{t=1}^{n} \xi_{t}^{* 2}+o_{p}^{*}(1)$, for which our proof is similar 
as Chang and Park (2002, Proof of Lemma 3.1(c)). Note that

$$
\begin{aligned}
\frac{1}{n} \sum_{t=1}^{n}\left(\xi_{p, t}^{*}-\xi_{t}^{*}\right)^{2} & =\frac{1}{n} \sum_{t=1}^{n}\left(\sum_{j=p+1}^{\infty} \hat{\Psi}_{p, j}^{\prime} \varepsilon_{t-j}^{*}\right)^{2} \\
& =\sum_{j=p+1}^{\infty} \sum_{i=p+1}^{\infty} \hat{\Psi}_{p, j}^{\prime}\left(\frac{1}{n} \sum_{t=1}^{n} \varepsilon_{t-j}^{*} \varepsilon_{t-i}^{* \prime}\right) \hat{\Psi}_{p, i} \\
& =\sum_{j=p+1}^{\infty} \hat{\Psi}_{p, j}^{\prime} \Sigma^{*} \hat{\Psi}_{j}+\sum_{j=p+1}^{\infty} \sum_{i=p+1}^{\infty}\left(\hat{\Psi}_{p, j}^{\prime}\left(\frac{1}{n} \sum_{t=1}^{n} \varepsilon_{t-j}^{*} \varepsilon_{t-i}^{* \prime}\right) \hat{\Psi}_{p, i}-\delta_{i j} \Sigma\right) \\
& =\sum_{j=p+1}^{\infty}\left|\hat{\Psi}_{p, j}\right|^{2} O_{p}^{*}(1)+\sum_{j=p+1}^{\infty} \sum_{i=p+1}^{\infty}\left|\hat{\Psi}_{p, j}\right|\left|\hat{\Psi}_{p, i}\right| O_{p}^{*}\left(n^{-1 / 2}\right)=o_{p}^{*}(1) .
\end{aligned}
$$

Then, as

$$
\left|\left(\frac{1}{n} \sum_{t=1}^{n} \xi_{p, t}^{* 2}\right)^{1 / 2}-\left(\frac{1}{n} \sum_{t=1}^{n} \xi_{t}^{* 2}\right)^{1 / 2}\right| \leq\left(\frac{1}{n} \sum_{t=1}^{n}\left(\xi_{p, t}^{*}-\xi_{t}^{*}\right)^{2}\right)^{1 / 2}
$$

as a consequence from Cauchy's inequality, ${ }^{9}$ it follows that

$$
\frac{1}{n} \sum_{t=1}^{n} \xi_{p, t}^{* 2}=\frac{1}{n} \sum_{t=1}^{n} \xi_{t}^{* 2}+o_{p}^{*}(1)
$$

which concludes this step.

For the final step, we first note that by the law of large numbers we have that

$$
\frac{1}{n} \sum_{t=1}^{n} \xi_{t}^{* 2} \stackrel{p^{*}}{\longrightarrow} \mathrm{E}^{*}\left(\xi_{t}^{2}\right)=\omega^{* 2}
$$

${ }^{9}$ From Cauchy's inequality we can derive that

$$
\begin{aligned}
& -\frac{2}{n}\left(\sum_{t=1}^{n} \xi_{p, t}^{* 2}\right)^{1 / 2}\left(\sum_{t=1}^{n} \xi_{t}^{* 2}\right)^{1 / 2} \leq-\frac{2}{n} \sum_{t=1}^{n} \xi_{p, t}^{*} \xi_{t}^{*} \\
\Leftrightarrow & \frac{1}{n} \sum_{t=1}^{n} \xi_{p, t}^{* 2}+\frac{1}{n} \sum_{t=1}^{n} \xi_{t}^{* 2}-2\left(\frac{1}{n} \sum_{t=1}^{n} \xi_{p, t}^{* 2}\right)^{1 / 2}\left(\frac{1}{n} \sum_{t=1}^{n} \xi_{t}^{* 2}\right)^{1 / 2} \leq \frac{1}{n} \sum_{t=1}^{n} \xi_{p, t}^{* 2}+\frac{1}{n} \sum_{t=1}^{n} \xi_{t}^{* 2}-2 \frac{1}{n} \sum_{t=1}^{n} \xi_{p, t}^{*} \xi_{t}^{*} \\
\Leftrightarrow & \left(\left(\frac{1}{n} \sum_{t=1}^{n} \xi_{p, t}^{* 2}\right)^{1 / 2}-\left(\frac{1}{n} \sum_{t=1}^{n} \xi_{t}^{* 2}\right)^{1 / 2}\right)^{2} \leq \frac{1}{n} \sum_{t=1}^{n}\left(\xi_{p, t}^{*}-\xi_{t}^{*}\right)^{2} \\
\Leftrightarrow & \left|\left(\frac{1}{n} \sum_{t=1}^{n} \xi_{p, t}^{* 2}\right)^{1 / 2}-\left(\frac{1}{n} \sum_{t=1}^{n} \xi_{t}^{* 2}\right)^{1 / 2}\right| \leq\left(\frac{1}{n} \sum_{t=1}^{n}\left(\xi_{p, t}^{*}-\xi_{t}^{*}\right)^{2}\right)^{1 / 2} .
\end{aligned}
$$


We then have to show that

$$
\omega^{* 2} \stackrel{p}{\rightarrow} \omega^{2} .
$$

As

$$
\omega^{* 2}=\sigma_{11}^{*}-\Sigma_{12}^{*} \Sigma_{22}^{*-1} \Sigma_{21}^{*} \quad \text { and } \quad \omega^{2}=\sigma_{11}-\Sigma_{12} \Sigma_{22}^{-1} \Sigma_{21},
$$

and $\Sigma^{*} \stackrel{p}{\rightarrow} \Sigma$ by Lemma 3, (44) follows. This completes the proof.

Proof of Theorem 3. Note that

$$
T^{*}=n \hat{\delta}^{* \prime}\left(n^{2} \widehat{\operatorname{Var}^{*}\left(\hat{\delta}^{*}\right)}\right)^{-1} n \hat{\delta}^{*} .
$$

We first look at $\hat{\delta}^{*}$. We can write $n \hat{\delta}^{*}$ as

$$
\begin{aligned}
n \hat{\delta}^{*} & =n\left[Z_{-1}^{* \prime}\left(I-W_{p}^{*}\left(W_{p}^{* \prime} W_{p}^{*}\right)^{-1} W_{p}^{* \prime}\right) Z_{-1}^{*}\right]^{-1} Z_{-1}^{* \prime}\left(I-W_{p}^{*}\left(W_{p}^{* \prime} W_{p}^{*}\right)^{-1} W_{p}^{* \prime}\right) \Delta Y^{*} \\
& =n\left[Z_{-1}^{* \prime}\left(I-W_{p}^{*}\left(W_{p}^{* \prime} W_{p}^{*}\right)^{-1} W_{p}^{* \prime}\right) Z_{-1}^{*}\right]^{-1} Z_{-1}^{* \prime}\left(I-W_{p}^{*}\left(W_{p}^{* \prime} W_{p}^{*}\right)^{-1} W_{p}^{* \prime}\right) \xi_{p}^{*} \\
& =\left[n^{-2} Z_{-1}^{* \prime} Z_{-1}^{*}-A_{n}^{*}\right]^{-1}\left(n^{-1} Z_{-1}^{* \prime} \xi_{p}^{*}-B_{n}^{*}\right),
\end{aligned}
$$

where

$$
A_{n}^{*}=n^{-2} Z_{-1}^{*} W_{p}^{*}\left(W_{p}^{* \prime} W_{p}^{*}\right)^{-1} W_{p}^{* \prime} Z_{-1}^{*}
$$

and

$$
B_{n}^{*}=n^{-1} Z_{-1}^{*} W_{p}^{*}\left(W_{p}^{* \prime} W_{p}^{*}\right)^{-1} W_{p}^{* \prime} \xi_{p}^{*}
$$

Using Lemma 6 c), d) and e), we have

$$
\begin{aligned}
\left|A_{n}^{*}\right| & \leq n^{-3}\left|Z_{-1}^{*} W_{p}^{*}\right|||\left(n^{-1} W_{p}^{* \prime} W_{p}^{*}\right)^{-1}||\left|W_{p}^{* \prime} Z_{-1}^{*}\right| \\
& =n^{-3} O_{p}^{*}\left(n p^{1 / 2}\right) O_{p}^{*}(1) O_{p}^{*}\left(n p^{1 / 2}\right)=O_{p}^{*}\left(n^{-1} p\right)
\end{aligned}
$$

and

$$
\begin{aligned}
\left|B_{n}^{*}\right| & \leq n^{-2}\left|Z_{-1}^{*} W_{p}^{*}\right|||\left(n^{-1} W_{p}^{* \prime} W_{p}^{*}\right)^{-1}||\left|W_{p}^{* \prime} \xi_{p}^{*}\right| \\
& =n^{-2} O_{p}^{*}\left(n p^{1 / 2}\right) O_{p}^{*}(1) O_{p}^{*}\left(n^{1 / 2} p^{1 / 2}\right)=O_{p}^{*}\left(n^{-1 / 2} p\right) .
\end{aligned}
$$

Hence, as $p=o\left(n^{1 / 2}\right)$ (Assumption 3), we have that

$$
A_{n}^{*}=o_{p}^{*}(1) \quad \text { and } \quad B_{n}^{*}=o_{p}^{*}(1) .
$$


Then by Lemma 6 a) and b), we have

$$
\begin{aligned}
n \hat{\delta}^{*} & =\left(n^{-2} Z_{-1}^{\prime} Z_{-1}\right)^{-1} n^{-1} Z_{-1}^{\prime} \xi_{p}+o_{p}^{*}(1) \\
& \stackrel{d^{*}}{\longrightarrow}\left[\int_{0}^{1} B(r) B(r)^{\prime} d r\right]^{-1} \int_{0}^{1} B(r) d B_{\xi}(r) .
\end{aligned}
$$

The estimated variance of $\hat{\delta}^{*}$, is defined as

$$
\left.\widehat{\operatorname{Var}^{*}\left(\hat{\delta}^{*}\right.}\right)=\hat{\omega}^{* 2}\left[Z_{-1}^{* \prime}\left(I-W_{p}^{*}\left(W_{p}^{* \prime} W_{p}^{*}\right)^{-1} W_{p}^{* \prime}\right) Z_{-1}^{*}\right]^{-1}
$$

Using Lemma 6 and Lemma 7, we have that

$$
\widehat{n^{2} \operatorname{Var}^{*}\left(\hat{\delta}^{*}\right)} \stackrel{d^{*}}{\longrightarrow} \omega^{2}\left[\int_{0}^{1} B(r) B(r)^{\prime} d r\right]^{-1} .
$$

Finally, using equations (45) and (46) we can derive that

$$
\begin{aligned}
T^{*} \stackrel{d^{*}}{\rightarrow} & \left(\int_{0}^{1} d B_{\xi}(r) B(r)^{\prime}\left[\int_{0}^{1} B(r) B(r)^{\prime} d r\right]^{-1}\right)\left(\omega^{2}\left[\int_{0}^{1} B(r) B(r)^{\prime} d r\right]^{-1}\right)^{-1} \\
& \times\left(\left[\int_{0}^{1} B(r) B(r)^{\prime} d r\right]^{-1} \int_{0}^{1} B(r) d B_{\xi}(r)\right) \\
= & \omega^{-2} \int_{0}^{1} d B_{\xi}(r) B(r)^{\prime}\left[\int_{0}^{1} B(r) B(r)^{\prime} d r\right]^{-1} \int_{0}^{1} B(r) d B_{\xi}(r) \\
= & \omega^{-2} \int_{0}^{1} d W_{1}(r) \omega W(r)^{\prime} L^{\prime} \Psi(1)^{\prime}\left[\int_{0}^{1} \Psi(1) L W(r) W(r)^{\prime} L^{\prime} \Psi(1)^{\prime} d r\right]^{-1} \\
& \times \int_{0}^{1} \Psi(1) L W(r) d W_{1}(r) \omega \\
= & \int_{0}^{1} d W_{1}(r) W(r)^{\prime}\left[\int_{0}^{1} W(r) W(r)^{\prime} d r\right]^{-1} \int_{0}^{1} W(r) d W_{1}(r)
\end{aligned}
$$

as $B(r)=\Psi(1) L W(r)$ and $B_{\xi}(r)=\omega W_{1}(r)$. This completes the proof.

\section{References}

Ahlgren, N. J. C. (2000). Bootstrapping the error correction model cointegration test. Working paper 428, Swedish School of Economics and Business Administration.

Banerjee, A., J. J. Dolado, and R. Mestre (1998). Error-correction mechanism tests for cointegration in a single-equation framework. Journal of Time Series Analysis 19, 267283.

Basawa, I. V., A. K. Mallik, W. P. McCormick, J. H. Reeves, and R. L. Taylor (1991). 
Bootstrapping unstable first-order autoregressive processes. Annals of Statistics 19, 10981101.

Berger, E. (1991). Majorization, exponential inequalities and almost sure behavior of vectorvalued random variables. Annals of Probability 19, 1206-1226.

Berk, K. N. (1974). Consistent autoregressive spectral estimates. Annals of Statistics 2, 489-502.

Boswijk, H. P. (1994). Testing for an unstable root in conditional and structural error correction models. Journal of Econometrics 63, 37-60.

Bühlmann, P. (1995). Moving-average representations of autoregressive approximations. Stochastic Processes and their Applications 60, 331-342.

Bühlmann, P. (1997). Sieve bootstrap for time series. Bernoulli 3, 123-148.

Chang, Y. and J. Y. Park (2002). On the asymptotics of ADF tests for unit roots. Econometric Reviews 21, 431-447.

Chang, Y. and J. Y. Park (2003). A sieve bootstrap for the test of a unit root. Journal of Time Series Analysis 24, 379-400.

Chang, Y., J. Y. Park, and K. Song (2006). Bootstrapping cointegrating regressions. Journal of Econometrics 133, 703-739.

De Acosta, A. (1981). Inequalities for $B$-valued random vectors with applications to the strong law of large numbers. Annals of Probability 9, 157-161.

Einmahl, U. (1987). A useful estimate in the multidimensional invariance principle. Probability Theory and Related Fields 76, 81-101.

Engle, R. F. and C. W. J. Granger (1987). Co-integration and error correction: representation, estimation and testing. Econometrica 55, 251-276.

Hannan, E. J. and L. Kavalieris (1986). Regression, autoregression models. Journal of Time Series Analysis 7, 27-49.

Johansen, S. (1995). Likelihood-Based Inference in Cointegrated Vector Autoregressive Models. Oxford: Oxford University Press.

Johansen, S. and K. Juselius (1990). Maximum likelihood estimation and inference on cointegration - with applications to the demand for money. Oxford Bulletin of Economics and Statistics 54, 169-210. 
Kremers, J. J. M., N. R. Ericsson, and J. J. Dolado (1992). The power of cointegration tests. Oxford Bulletin of Economics and Statistics 52, 325-348.

Mantalos, P. and G. Shukur (1998). Size and power of the error correction model cointegration test. A bootstrap approach. Oxford Bulletin of Economics and Statistics 60, 249-255.

Móricz, F. (1976). Moment inequalities and the strong law of large numbers. Zeitschrift für Wahrscheinlichkeitstheorie und verwandte Gebiete 35, 299-314.

Palm, F. C., S. Smeekes, and J.-P. Urbain (2007). Bootstrap unit root tests: comparison and extensions. Journal of Time Series Analysis, forthcoming.

Paparoditis, E. (1996). Bootstrapping autoregressive and moving average parameter estimates of infinite order vector autoregressive processes. Journal of Multivariate Analysis 57, 277296.

Paparoditis, E. and D. N. Politis (2005). Bootstrapping unit root tests for autoregressive time series. Journal of the American Statistical Association 100, 545-553.

Park, J. Y. (2002). An invariance principle for sieve bootstrap in time series. Econometric Theory 18, 469-490.

Park, J. Y. and P. C. B. Phillips (1989). Statistical inference in regressions with integrated processes: part 2. Econometric Theory 5, 95-131.

Pesavento, E. (2004). Analytical evaluation of the power of tests for the absence of cointegration. Journal of Econometrics 122, 349-384.

Phillips, P. C. B. (1988). Weak convergence to the matrix stochastic integral $\int_{0}^{1} B d B^{\prime}$. Journal of Multivariate Analysis 24, 252-264.

Phillips, P. C. B. and V. Solo (1992). Asymptotics for linear processes. Annals of Statistics 20, 971-1001.

Seo, M. (2006). Bootstrap testing for the null of no cointegration in a threshold vector error correction model. Journal of Econometrics 134, 129-150.

Swensen, A. R. (2006). Bootstrap algorithms for testing and determining the cointegration rank in VAR models. Econometrica 74, 1699-1714.

Trenkler, C. (2006). Bootstrapping systems cointegration tests with a prior adjustment for deterministic terms. SFB 649 Discussion Paper 2006-012, Humboldt Universität zu Berlin.

Van Giersbergen, N. P. A. and J. F. Kiviet (1996). Bootstrapping a stable AD model: weak vs strong exogeneity. Oxford Bulletin of Economics and Statistics 58, 631-656. 
Zivot, E. (2000). The power of single equation tests for cointegration when the cointegrating vector is prespecified. Econometric Theory 16, 407-439. 
Table 1: Parameter combinations used in the simulation DGP

\begin{tabular}{|c|c|c|c|}
\hline$\Phi$ & $\Theta$ & $r$ & $c$ \\
\hline $\begin{array}{ll}0 & 0\end{array}$ & $\left.\begin{array}{|ll}0 & 0\end{array}\right]$ & \multirow{2}{*}{$0, \sqrt{0.3}, \sqrt{0.7}$} & \multirow{2}{*}{$0,-5,-10,-20$} \\
\hline $\begin{array}{ll}0 & 0\end{array}$ & $\begin{array}{ll}0 & 0\end{array}$ & & \\
\hline$\left[\begin{array}{ll}0.2 & 0\end{array}\right.$ & {$\left[\begin{array}{ll}0 & 0\end{array}\right]$} & \multirow{2}{*}{$\sqrt{0.3}$} & \multirow{2}{*}{0} \\
\hline $\begin{array}{ll}0 & 0.2\end{array}$ & $\begin{array}{ll}0 & 0\end{array}$ & & \\
\hline $0.8 \quad 0$ & {$\left[\begin{array}{ll}0 & 0\end{array}\right]$} & \multirow{2}{*}{$\sqrt{0.3}$} & \multirow{2}{*}{0} \\
\hline $\begin{array}{ll}0 & 0.8\end{array}$ & $\begin{array}{ll}0 & 0\end{array}$ & & \\
\hline$\left[\begin{array}{ll}0.2 & 0.5\end{array}\right]$ & 0 & \multirow{2}{*}{$\sqrt{0.3}$} & \multirow{2}{*}{$0,-5,-10,-20$} \\
\hline $0.5 \quad 0.2$ & {$\left[\begin{array}{ll}0 & 0\end{array}\right]$} & & \\
\hline$\left[\begin{array}{ll}0 & 0\end{array}\right]$ & {$\left[\begin{array}{ll}0.2 & 0\end{array}\right]$} & \multirow{2}{*}{$\sqrt{0.3}$} & \multirow{2}{*}{0} \\
\hline$\left[\begin{array}{ll}0 & 0\end{array}\right]$ & {$\left[\begin{array}{ll}0 & 0.2\end{array}\right]$} & & \\
\hline$\left[\begin{array}{ll}0 & 0\end{array}\right]$ & {$\left[\begin{array}{ll}0.8 & 0\end{array}\right]$} & \multirow{2}{*}{$\sqrt{0.3}$} & \multirow{2}{*}{0} \\
\hline$\left[\begin{array}{ll}0 & 0\end{array}\right]$ & 0.8 & & \\
\hline$\left[\begin{array}{ll}0 & 0\end{array}\right]$ & {$\left[\begin{array}{ll}0.2 & 0.5\end{array}\right]$} & \multirow{2}{*}{$\sqrt{0.3}$} & \multirow{2}{*}{$0,-5,-10,-20$} \\
\hline 0 & 0.2 & & \\
\hline$\left[\begin{array}{ll}0 & 0\end{array}\right]$ & {$[-0.8$} & \multirow{2}{*}{$\sqrt{0.3}$} & \multirow{2}{*}{0} \\
\hline$\left[\begin{array}{ll}0 & 0\end{array}\right]$ & -0.8 & & \\
\hline
\end{tabular}


Table 2: Size and power for white noise errors

\begin{tabular}{|c|c|c|c|c|c|c|c|}
\hline$r$ & $c$ & $T_{v, n}^{*}$ & $T_{v, a}^{*}$ & $T_{c, n}^{*}$ & $T_{c, a}^{*}$ & $T_{a s}$ & $T_{s c}$ \\
\hline \multicolumn{8}{|c|}{$n=50$} \\
\hline \multirow[t]{4}{*}{0} & 0 & 0.042 & 0.042 & 0.043 & 0.040 & 0.072 & 0.050 \\
\hline & -5 & 0.102 & 0.109 & 0.105 & 0.103 & 0.163 & 0.121 \\
\hline & -10 & 0.281 & 0.283 & 0.284 & 0.283 & 0.414 & 0.322 \\
\hline & -20 & 0.831 & 0.839 & 0.829 & 0.835 & 0.918 & 0.866 \\
\hline \multirow[t]{4}{*}{$\sqrt{0.3}$} & 0 & 0.052 & 0.050 & 0.052 & 0.051 & 0.085 & 0.050 \\
\hline & -5 & 0.163 & 0.159 & 0.160 & 0.162 & 0.243 & 0.163 \\
\hline & -10 & 0.521 & 0.523 & 0.522 & 0.522 & 0.661 & 0.524 \\
\hline & -20 & 0.949 & 0.961 & 0.950 & 0.960 & 0.984 & 0.964 \\
\hline \multirow[t]{4}{*}{$\sqrt{0.7}$} & 0 & 0.052 & 0.051 & 0.051 & 0.052 & 0.079 & 0.050 \\
\hline & -5 & 0.501 & 0.505 & 0.498 & 0.503 & 0.618 & 0.488 \\
\hline & -10 & 0.898 & 0.901 & 0.898 & 0.903 & 0.935 & 0.898 \\
\hline & -20 & 0.983 & 0.996 & 0.985 & 0.997 & 0.999 & 0.998 \\
\hline \multicolumn{8}{|c|}{$n=100$} \\
\hline \multirow[t]{4}{*}{0} & 0 & 0.048 & 0.048 & 0.048 & 0.049 & 0.059 & 0.050 \\
\hline & -5 & 0.108 & 0.107 & 0.108 & 0.109 & 0.133 & 0.113 \\
\hline & -10 & 0.317 & 0.320 & 0.315 & 0.314 & 0.381 & 0.334 \\
\hline & -20 & 0.868 & 0.859 & 0.865 & 0.861 & 0.906 & 0.875 \\
\hline \multirow[t]{4}{*}{$\sqrt{0.3}$} & 0 & 0.061 & 0.057 & 0.061 & 0.059 & 0.072 & 0.050 \\
\hline & -5 & 0.180 & 0.187 & 0.181 & 0.185 & 0.225 & 0.160 \\
\hline & -10 & 0.541 & 0.545 & 0.535 & 0.541 & 0.601 & 0.501 \\
\hline & -20 & 0.960 & 0.961 & 0.963 & 0.963 & 0.978 & 0.952 \\
\hline \multirow[t]{4}{*}{$\sqrt{0.7}$} & 0 & 0.056 & 0.056 & 0.053 & 0.058 & 0.071 & 0.050 \\
\hline & -5 & 0.545 & 0.543 & 0.538 & 0.539 & 0.597 & 0.524 \\
\hline & -10 & 0.936 & 0.936 & 0.938 & 0.933 & 0.949 & 0.933 \\
\hline & -20 & 0.999 & 1.000 & 1.000 & 1.000 & 1.000 & 1.000 \\
\hline
\end{tabular}


Table 3: Size for serially correlated errors

\begin{tabular}{|c|c|c|c|c|c|c|c|c|}
\hline \multicolumn{2}{|c|}{$\Phi$} & \multicolumn{2}{|c|}{$\Theta$} & $T_{v, n}^{*}$ & $\overline{T_{v, a}^{*}}$ & $T_{c, n}^{*}$ & $T_{c, a}^{*}$ & $T_{a s}$ \\
\hline \multicolumn{9}{|c|}{$n=50$} \\
\hline$\left[\begin{array}{c}0.2 \\
0\end{array}\right.$ & $\left.\begin{array}{c}0 \\
0.2\end{array}\right]$ & {$\left[\begin{array}{l}0 \\
0\end{array}\right.$} & $\left.\begin{array}{l}0 \\
0\end{array}\right]$ & 0.060 & 0.057 & 0.059 & 0.058 & 0.095 \\
\hline $\begin{array}{c}0.8 \\
0\end{array}$ & $\left.\begin{array}{c}0 \\
0.8\end{array}\right]$ & $\begin{array}{l}0 \\
0 \\
0\end{array}$ & $\begin{array}{l}0 \\
0\end{array}$ & 0.049 & 0.045 & 0.045 & 0.046 & 0.158 \\
\hline$\left[\begin{array}{l}0.2 \\
0.5\end{array}\right.$ & $\left.\begin{array}{l}0.5 \\
0.2\end{array}\right]$ & {$\left[\begin{array}{l}0 \\
0\end{array}\right.$} & $\left.\begin{array}{l}0 \\
0\end{array}\right]$ & 0.059 & 0.088 & 0.055 & 0.088 & 0.214 \\
\hline$\left[\begin{array}{l}0 \\
0\end{array}\right.$ & $\left.\begin{array}{l}0 \\
0\end{array}\right]$ & {$\left[\begin{array}{c}0.2 \\
0\end{array}\right.$} & $\left.\begin{array}{c}0 \\
0.2\end{array}\right]$ & 0.063 & 0.061 & 0.065 & 0.058 & 0.103 \\
\hline$\left[\begin{array}{l}0 \\
0\end{array}\right.$ & $\begin{array}{l}0 \\
0\end{array}$ & $\begin{array}{c}0.8 \\
0\end{array}$ & $\left.\begin{array}{c}0 \\
0.8\end{array}\right]$ & 0.050 & 0.055 & 0.050 & 0.055 & 0.186 \\
\hline$\left[\begin{array}{l}0 \\
0\end{array}\right.$ & $\begin{array}{l}0 \\
0\end{array}$ & {$\left[\begin{array}{l}0.2 \\
0.5\end{array}\right.$} & $\left.\begin{array}{l}0.5 \\
0.2\end{array}\right]$ & 0.075 & 0.092 & 0.075 & 0.095 & 0.188 \\
\hline$\left[\begin{array}{l}0 \\
0\end{array}\right.$ & $\left.\begin{array}{l}0 \\
0\end{array}\right]$ & {$\left[\begin{array}{c}-0.8 \\
0\end{array}\right.$} & $\left.\begin{array}{c}0 \\
-0.8\end{array}\right]$ & 0.459 & 0.625 & 0.453 & 0.625 & 0.677 \\
\hline \multicolumn{9}{|c|}{$n=100$} \\
\hline$\left[\begin{array}{c}0.2 \\
0\end{array}\right.$ & $\left.\begin{array}{c}0 \\
0.2\end{array}\right]$ & {$\left[\begin{array}{l}0 \\
0\end{array}\right.$} & $\left.\begin{array}{l}0 \\
0\end{array}\right]$ & 0.063 & 0.065 & 0.059 & 0.064 & 0.084 \\
\hline $\begin{array}{c}0.8 \\
0\end{array}$ & $\left.\begin{array}{c}0 \\
0.8\end{array}\right]$ & {$\left[\begin{array}{l}0 \\
0\end{array}\right.$} & $\begin{array}{l}0 \\
0\end{array}$ & 0.051 & 0.047 & 0.050 & 0.049 & 0.091 \\
\hline$\left[\begin{array}{l}0.2 \\
0.5\end{array}\right.$ & $\left.\begin{array}{l}0.5 \\
0.2\end{array}\right]$ & {$\left[\begin{array}{l}0 \\
0\end{array}\right.$} & $\begin{array}{l}0 \\
0\end{array}$ & 0.056 & 0.056 & 0.056 & 0.052 & 0.107 \\
\hline$\left[\begin{array}{l}0 \\
0\end{array}\right.$ & $\left.\begin{array}{l}0 \\
0\end{array}\right]$ & {$\left[\begin{array}{c}0.2 \\
0\end{array}\right.$} & $\left.\begin{array}{c}\rfloor \\
0.2\end{array}\right]$ & 0.059 & 0.059 & 0.059 & 0.058 & 0.080 \\
\hline$\left[\begin{array}{l}0 \\
0\end{array}\right.$ & $\left.\begin{array}{l}0 \\
0\end{array}\right]$ & {$\left[\begin{array}{c}0.8 \\
0\end{array}\right.$} & $\left.\begin{array}{c}0 \\
0.8\end{array}\right]$ & 0.057 & 0.067 & 0.058 & 0.066 & 0.120 \\
\hline$\left[\begin{array}{l}0 \\
0\end{array}\right.$ & $\left.\begin{array}{l}0 \\
0\end{array}\right]$ & {$\left[\begin{array}{l}0.2 \\
0.5\end{array}\right.$} & $\left.\begin{array}{l}0.5 \\
0.2\end{array}\right]$ & 0.070 & 0.075 & 0.067 & 0.078 & 0.140 \\
\hline$\left[\begin{array}{l}0 \\
0\end{array}\right.$ & $\left.\begin{array}{l}0 \\
0\end{array}\right]$ & {$\left[\begin{array}{c}-0.8 \\
0\end{array}\right.$} & $\left.\begin{array}{c}0 \\
-0.8\end{array}\right]$ & 0.485 & 0.518 & 0.481 & 0.525 & 0.611 \\
\hline
\end{tabular}


Table 4: Power for serially correlated errors

\begin{tabular}{|c|c|c|c|c|c|c|c|c|c|}
\hline \multicolumn{3}{|c|}{ dynamics } & $c$ & $T_{v, n}^{*}$ & $T_{v, a}^{*}$ & $T_{c, n}^{*}$ & $T_{c, a}^{*}$ & $T_{a s}$ & $T_{s c}$ \\
\hline \multicolumn{10}{|c|}{$n=50$} \\
\hline \multirow{3}{*}{$\Phi=$} & \multirow{3}{*}{$\begin{array}{l}0.2 \\
0.5\end{array}$} & \multirow{3}{*}{$\left.\begin{array}{l}0.5 \\
0.2\end{array}\right]$} & -5 & 0.624 & 0.538 & 0.625 & 0.539 & 0.819 & 0.485 \\
\hline & & & -10 & 0.857 & 0.844 & 0.856 & 0.845 & 0.964 & 0.735 \\
\hline & & & -20 & 0.929 & 0.978 & 0.930 & 0.978 & 0.996 & 0.899 \\
\hline \multirow{3}{*}{$\Theta=$} & \multirow{3}{*}{$\begin{array}{l}0.2 \\
0.5\end{array}$} & \multirow{3}{*}{$\left.\begin{array}{l}0.5 \\
0.2\end{array}\right]$} & -5 & 0.401 & 0.301 & 0.411 & 0.298 & 0.604 & 0.259 \\
\hline & & & -10 & 0.810 & 0.749 & 0.818 & 0.753 & 0.939 & 0.650 \\
\hline & & & -20 & 0.940 & 0.968 & 0.942 & 0.969 & 0.994 & 0.957 \\
\hline \multicolumn{10}{|c|}{$n=100$} \\
\hline \multirow{3}{*}{$\Phi=$} & \multirow{3}{*}{$\begin{array}{l}0.2 \\
0.5\end{array}$} & \multirow{3}{*}{$\left.\begin{array}{l}0.5 \\
0.2\end{array}\right]$} & -5 & 0.893 & 0.895 & 0.898 & 0.896 & 0.948 & 0.891 \\
\hline & & & -10 & 0.993 & 0.989 & 0.993 & 0.988 & 0.996 & 0.987 \\
\hline & & & -20 & 0.999 & 1.000 & 0.999 & 1.000 & 1.000 & 1.000 \\
\hline \multirow{3}{*}{$\Theta=$} & \multirow{3}{*}{$\begin{array}{l}0.2 \\
0.5\end{array}$} & \multirow{3}{*}{$\left.\begin{array}{l}0.5 \\
0.2\end{array}\right]$} & -5 & 0.493 & 0.470 & 0.491 & 0.473 & 0.681 & 0.416 \\
\hline & & & -10 & 0.907 & 0.889 & 0.905 & 0.884 & 0.973 & 0.851 \\
\hline & & & -20 & 0.998 & 0.998 & 0.996 & 0.996 & 1.000 & 0.996 \\
\hline
\end{tabular}


Table 5: Size and power for tests with deterministic trends

\begin{tabular}{|c|c|c|c|c|c|c|c|c|c|c|c|}
\hline $\begin{array}{l}\mu_{1} \\
\mu_{2} \\
\end{array}$ & & $D_{t}^{(r)}$ & $T_{v, n}^{*}$ & $T_{v, a}^{*}$ & $T_{a s}$ & $T_{v, n}^{*}$ & $T_{v, a}^{*}$ & $T_{a s}$ & $T_{v, n}^{*}$ & $T_{v, a}^{*}$ & $T_{a s}$ \\
\hline & & & \multicolumn{3}{|c|}{$\Phi=\Theta=0$} & $\Phi=$ & {$\left[\begin{array}{l}0.2 \\
0.5\end{array}\right.$} & 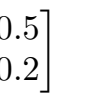 & $\Theta=$ & $=\left[\begin{array}{l}0.2 \\
0.5\end{array}\right.$ & $\left.\begin{array}{l}0.5 \\
0.2\end{array}\right]$ \\
\hline \multicolumn{12}{|c|}{$c=0$} \\
\hline & & $D_{t}^{r}=1$ & 0.043 & 0.040 & 0.119 & 0.044 & 0.112 & 0.298 & 0.062 & 0.088 & 0.246 \\
\hline 0 & 0 & $D_{t}=1$ & 0.045 & 0.048 & 0.097 & 0.043 & 0.086 & 0.255 & 0.066 & 0.106 & 0.245 \\
\hline \multirow{3}{*}{\multicolumn{2}{|c|}{0}} & $D_{t}^{r}=t$ & 0.051 & 0.046 & 0.163 & 0.042 & 0.085 & 0.413 & 0.053 & 0.079 & 0.375 \\
\hline & & $D_{t}^{\prime}=1, t$ & 0.044 & 0.042 & 0.153 & 0.043 & 0.099 & 0.384 & 0.047 & 0.072 & 0.350 \\
\hline & & $D_{t}^{r}=1$ & 0.047 & 0.046 & 0.114 & 0.042 & 0.110 & 0.303 & 0.061 & 0.082 & 0.239 \\
\hline 1 & 0 & $D_{t}=1$ & 0.044 & 0.048 & 0.099 & 0.045 & 0.101 & 0.270 & 0.069 & 0.102 & 0.246 \\
\hline \multirow[t]{2}{*}{1} & 0 & $D_{t}^{r}=t$ & 0.050 & 0.044 & 0.164 & 0.043 & 0.086 & 0.407 & 0.059 & 0.075 & 0.353 \\
\hline & & $D_{t}^{\prime}=1, t$ & 0.050 & 0.051 & 0.158 & 0.047 & 0.089 & 0.373 & 0.066 & 0.091 & 0.370 \\
\hline 1 & 0 & $D_{t}^{r}=1$ & 0.044 & 0.043 & 0.147 & 0.043 & 0.094 & 0.409 & 0.056 & 0.080 & 0.358 \\
\hline 1 & 1 & $D_{t}^{\prime}=1, t$ & 0.043 & 0.043 & 0.159 & 0.035 & 0.081 & 0.378 & 0.061 & 0.080 & 0.354 \\
\hline 1 & 1 & $D_{t}^{r}=1$ & 0.043 & 0.043 & 0.173 & 0.046 & 0.092 & 0.403 & 0.051 & 0.079 & 0.391 \\
\hline 1 & 1 & $D_{t}^{\prime}=1, t$ & 0.047 & 0.044 & 0.161 & 0.043 & 0.095 & 0.375 & 0.057 & 0.085 & 0.355 \\
\hline \multicolumn{12}{|c|}{$c=-10$} \\
\hline & & $D_{t}^{r}=1$ & 0.208 & 0.212 & 0.437 & 0.591 & 0.545 & 0.891 & 0.484 & 0.361 & 0.803 \\
\hline 0 & 0 & $D_{t}=1$ & 0.239 & 0.248 & 0.450 & 0.608 & 0.534 & 0.891 & 0.516 & 0.384 & 0.798 \\
\hline \multirow{3}{*}{\multicolumn{2}{|c|}{0}} & $D_{t}^{r}=t$ & 0.083 & 0.086 & 0.404 & 0.267 & 0.199 & 0.836 & 0.233 & 0.118 & 0.707 \\
\hline & & $D_{t}^{\prime}=1, t$ & 0.094 & 0.095 & 0.407 & 0.301 & 0.222 & 0.820 & 0.258 & 0.147 & 0.708 \\
\hline & & $D_{t}^{r}=1$ & 0.206 & 0.206 & 0.425 & 0.597 & 0.527 & 0.887 & 0.503 & 0.352 & 0.811 \\
\hline 1 & 0 & $D_{t}=1$ & 0.244 & 0.246 & 0.450 & 0.610 & 0.540 & 0.875 & 0.518 & 0.386 & 0.799 \\
\hline \multirow{2}{*}{\multicolumn{2}{|c|}{1}} & $D_{t}^{r}=t$ & 0.091 & 0.084 & 0.383 & 0.290 & 0.232 & 0.850 & 0.235 & 0.107 & 0.711 \\
\hline & & $D_{t}^{\prime}=1, t$ & 0.092 & 0.098 & 0.407 & 0.295 & 0.216 & 0.803 & 0.252 & 0.126 & 0.716 \\
\hline 1 & 0 & $D_{t}^{r}=t$ & 0.086 & 0.091 & 0.391 & 0.287 & 0.218 & 0.829 & 0.249 & 0.126 & 0.705 \\
\hline 1 & 1 & $D_{t}^{\prime}=1, t$ & 0.110 & 0.112 & 0.423 & 0.315 & 0.220 & 0.816 & 0.263 & 0.144 & 0.721 \\
\hline 1 & 1 & $D_{t}^{r}=t$ & 0.090 & 0.082 & 0.392 & 0.279 & 0.215 & 0.830 & 0.242 & 0.131 & 0.720 \\
\hline 1 & 1 & $D_{t}^{\prime}=1, t$ & 0.105 & 0.106 & 0.414 & 0.309 & 0.231 & 0.806 & 0.242 & 0.120 & 0.713 \\
\hline
\end{tabular}

